ALEA, Lat. Am. J. Probab. Math. Stat. 14, 445-471 (2017)

DOI: 10.30757/ALEA.v14-24

\title{
Self-exciting piecewise linear processes
}

\section{Nikita Ratanov}

Facultad de Economía, Universidad del Rosario,

Calle 12c, No.4-69

Bogotá, Colombia

E-mail address: nikita.ratanov@urosario.edu.co

URL: http://www.urosario.edu.co/Profesores/Listado-de-profesores/R/Nikita-Ratanov

\begin{abstract}
The paper concerns a piecewise linear process controlled by the set of velocities $\left\{c_{n}\right\}_{n \geq 0}$ consecutively switched after exponentially distributed, $\operatorname{Exp}\left(\lambda_{n}\right)$, $n \geq 0$, time intervals. The distribution of such process is studied in detail, including the distribution of the first passage time through the constant boundary.

The processes which moves by alternating patterns and with a double jump component are also studied.
\end{abstract}

\section{Introduction}

A class of random processes with excitation at random times is studied for a long time in various aspects. The main example of the process of that manner of modeling is the telegraph process. On the physical parlance such process is also called "steady-state random walks". The telegraph processes describing noninteracting particles, which move with finite constant velocities switchable over an exponentially distributed random time intervals have been studied by many authors beginning with Taylor (1922).

This model with the alternating velocities $\pm c$ has been later developed by Goldstein (1951) in connection with hyperbolic partial differential equations. In 1956 Mark Kac began to study the telegraph model in detail, see Kac (1974). The distribution density $p=p(x, t)$ of the particles' positions follows the telegraph (damped wave) equation

$$
\frac{\partial^{2} p}{\partial t^{2}}+2 \lambda \frac{\partial p}{\partial t}=c^{2} \frac{\partial^{2} p}{\partial x^{2}}
$$

where $\lambda$ is the switching intensity.

Afterwards, the telegraph process has been studied in great detail with many generalisations. In particular, the motions with the velocities alternating in gamma-

Received by the editors July 19th, 2016; accepted May 6th, 2017.

2010 Mathematics Subject Classification. 60J75, 60K15.

Key words and phrases. piecewise linear process, steady-state random walk, jump-telegraph process, first passage time, martingales. 
or Erlang-distributed random intervals have been studied many times, see e. g. Di Crescenzo (2001); Zacks (2004); Di Crescenzo and Martinucci (2010). Motions characterised by more then two possible deterministic velocities (Orsingher and Bassan, 1992; Kolesnik, 1998; Samollenko, 2002) or random velocities (Stadje and Zacks, 2004; López and Ratanov, 2012) have been also considered.

Here we present the similar processes $L=L(t), t \geq 0$, with the sequential velocities, $\left\{c_{n}\right\}_{n \geq 0}$, switched by the successive intensities $\left\{\lambda_{n}\right\}_{n \geq 0}$. The model is based on piecewise-deterministic Markov processes which originally have been designed for queueing theory, see Gnedenko and Kovalenko (1968). These models are used in various fields, see Costa and Davis (1989); Davis (1984); de Saporta and Dufour (2012). Instead of PDE (1.1), by conditioning on the first switching one can easily get integral equations for distributions of these processes, see equations (2.10) and (3.13). However, a detailed analysis of the distributions of these processes still was not done.

These processes can be considered as an example of self-exciting Hawkes processes well-studied before, beginning with the seminal paper by Hawkes (1971). Recently the Hawkes processes are intensively applied in finance, see e.g. Embrechts et al. (2011). See also an overview by Bacry et al. (2015). In the context of market model based on Hawkes processes the optimal execution problem is solved explicitly by Alfonsi and Blanc (2016).

This presentation continues the author's paper Ratanov (2014), where the explicit formula for the moment generating function of the piecewise linear process $L(t), t>0$, was given. The distributions of these processes are studied in Section 2 , including the distribution of the first passage time $T(x)$ (for the process with positive velocities, where the level $x$ is also positive, see Section 2.2). In Section 2.3 we derive explicit formulae for the expectations of $L(t)$ with jumps $r_{n}$, occurring after each velocity switching.

Section 3 presents the piecewise linear processes, which are developing in accordance with alternately changing patterns. These processes are supplied with a double jump component. First, there is a jump immediately after each velocity switching (during the current pattern). Second, a jump accompanies the patterns changing. In the latter case a jump amplitude depends on the number of the velocity switchings during the current pattern. The system of integral equations for expectations of such process $X=X(t)$ is obtained. Further, there are described martingales of this form.

Telegraph-like processes have multiple applications including the applications to financial market modelling, see Di Mazi et al. (1994), and then, Ratanov (1999); Di Crescenzo and Pellerey (2002). Nowadays, these applications became the theory of Markov-modulated market models based on telegraph processes with alternating velocities, see e. g. Ratanov (2007, 2010); López and Ratanov (2014) (see also the survey in Kolesnik and Ratanov, 2013).

It has long been clear that for the purpose of financial modelling a simple replacement of a Wiener process on a telegraph usually produces arbitrage opportunities since the (stochastic) exponential of a telegraph process is not a martingale. To resolve the problem, one can study the model with jump and/or diffusion component, Kolesnik and Ratanov (2013); Ratanov (2010). However, erroneous models based only on the telegraph process continue to appear in publications, see e.g. De Gregorio (2016, Section 4). 
A sketch of the financial market model based on the double-jump process with alternately changing piecewise linear patterns is presented in Section 4. This model involves switching tendencies (accompanied by jumps of random amplitude) due to the internal market forces (small investors). Meanwhile, switching between the patterns is determined by a regulator policy (or an institutional/strategic investor). It turns out that a certain policy of the strategic investor could provoke arbitrage.

\section{Jump-telegraph processes with distinct parameters}

We repeatedly use the following notations.

Let $\vec{c}=\left\{c_{n}\right\}_{n \geq 0}$ and $\vec{\lambda}=\left\{\lambda_{n}\right\}_{n \geq 0}$ be two numerical sequences. Assume that all pairs $\left\langle c_{n}, \lambda_{n}\right\rangle, n \geq 0$, are distinct, i. e., if for $l \neq k, c_{l}=c_{k}$, then $\lambda_{l} \neq \lambda_{k}$. The products

$$
\begin{gathered}
\kappa_{n, k}(\vec{c}, \vec{\lambda})=\prod_{l=0, c_{l} \neq c_{k}}^{n}\left(c_{l}-c_{k}\right)^{-1} \times \prod_{\substack{l=0, c_{l}=c_{k} \\
l \neq k}}^{n}\left(\lambda_{l}-\lambda_{k}\right)^{-1}, \\
n \geq 1,0 \leq k \leq n,
\end{gathered}
$$

are well-defined; $\kappa_{0,0}=1$. If all $c_{n}$ are distinct, $c_{k} \neq c_{n}$ for $k \neq n$, then we use the shortened notation,

$$
\kappa_{n, k}(\vec{c})=\prod_{j=0, j \neq k}^{n}\left(c_{j}-c_{k}\right)^{-1}, \quad n \geq 1,0 \leq k \leq n .
$$

Note that due to the known Vandermonde properties, for $n \geq 1$

$$
\sum_{k=0}^{n} \kappa_{n, k}(\vec{c}) \frac{c_{k}^{m}}{\left(z+c_{k}\right)}=\frac{z^{m}}{\Pi_{n}(z)}, \quad 0 \leq m \leq n,
$$

(if $c_{k} \neq c_{n}$ for $k \neq n$ ), where $\Pi_{n}(z)=\Pi_{n}(z ; \vec{c})=\prod_{k=0}^{n}\left(z+c_{k}\right)$. Hence,

$$
\sum_{k=0}^{n} \kappa_{n, k}(\vec{c}) c_{k}^{m}=0, \quad 0 \leq m \leq n-1 .
$$

Moreover,

$$
\sum_{k=0}^{n} \kappa_{n, k}(\vec{c}) c_{k}^{n}=(-1)^{n},
$$

Kuznetsov (2003, p.11), see also Ratanov (2014, Remark 1 and (2.8)-(2.9)).

In what follows we assume $\sum_{\mathcal{A}} \cdot=0$ and $\prod_{\mathcal{A}} \cdot=1$ if $\mathcal{A}=\emptyset$.

2.1. Piecewise linear process. In this paper we study the continuous piecewise linear process $L=L(t), t \geq 0$,

$$
\begin{aligned}
L(t)=\int_{0}^{t} c_{N(u)} \mathrm{d} u & =\sum_{n=0}^{N(t)-1} c_{n} \tau_{n}+c_{N(t)}\left(t-\tau^{+, N(t)}\right) \\
& =\sum_{n=0}^{N(t)-1}\left(c_{n}-c_{N(t)}\right) \tau_{n}+c_{N(t)} t,
\end{aligned}
$$

which corresponds the position of a particle moving with successive velocities $c_{n}$, switching after elapsed times $\tau_{n}$. Here $\tau^{+, n}$ denotes the switching instants, $\tau^{+, n}=$ $\sum_{k=0}^{n-1} \tau_{k}, n \geq 1, \tau^{+, 0}=0$, and $N=N(t)$ is the corresponding counting process. 
Let $\pi(t ; n), n \geq 0$, be the probability mass function, $\pi(t ; n)=\mathbb{P}\{N(t)=n\}$. The expectation of $L(t), t \geq 0$, can be expressed by

$$
\mathbb{E}[L(t)]=\int_{0}^{t} \mathbb{E}\left[c_{N(u)}\right] \mathrm{d} u=\sum_{n=0}^{\infty} c_{n} \int_{0}^{t} \pi(u ; n) \mathrm{d} u,
$$

if the series converges.

Let $\tau_{n}$ be independent and exponentially distributed, $\tau_{n} \sim \operatorname{Exp}\left(\lambda_{n}\right), \lambda_{n}>0$, $n \geq 0$.

The probability mass functions $\pi(t ; n)=\mathbb{P}\{N(t)=n\}$ satisfy the system,

$$
\frac{\mathrm{d} \pi(t ; n)}{\mathrm{d} t}=-\lambda_{n} \pi(t ; n)+\lambda_{n-1} \pi(t ; n-1), \quad t>0 ; \quad n \geq 1
$$

and $\pi(t ; 0)=\mathrm{e}^{-\lambda_{0} t}, t \geq 0$, see Feller (1950).

Random variables $\tau^{+, n}, n \geq 1$, are Erlang-distributed. If all $\lambda^{\prime}$ s are distinct, then the density function $\varphi_{n}(t)$ of $\tau^{+, n}$ is given by

$$
\varphi_{n}(t)=\Lambda_{n} \sum_{k=0}^{n-1} \kappa_{n-1, k}(\vec{\lambda}) \mathrm{e}^{-\lambda_{k} t} \mathbf{1}_{\{t>0\}}, \quad n \geq 1,
$$

Here $\Lambda_{n}=\prod_{n-1}(0 ; \vec{\lambda})=\prod_{k=0}^{n-1} \lambda_{k}, n \geq 1, \Lambda_{0}=1$, and $\kappa_{n-1, k}(\vec{\lambda})$ are defined by (2.2). The usual modifications can be applied if two or more $\lambda_{k}$ are equal. For instance, $\varphi_{n}(t)=\frac{\lambda^{n} t^{n-1}}{(n-1) !} \mathrm{e}^{-\lambda t} \mathbf{1}_{\{t>0\}}$, if $\lambda_{n} \equiv \lambda, n \geq 0$.

If all $\lambda^{\prime}$ s are distinct, then by (2.7) the probability mass function of $N(t), t>0$, is given by

$$
\begin{aligned}
\pi(t ; n)=\mathbb{P}\{N(t)=n\} & =\mathbb{P}\left\{\tau^{+, n+1}>t\right\}-\mathbb{P}\left\{\tau^{+, n}>t\right\} \\
& =\Lambda_{n} \sum_{k=0}^{n} \kappa_{n, k}(\vec{\lambda}) \mathrm{e}^{-\lambda_{k} t}, \quad n \geq 0
\end{aligned}
$$

with the usual modifications, if some of $\lambda_{k}$ are equal. Cf. Ratanov (2014, (2.4)).

It's known that process $N$ is non-explosive, $\mathbb{P}\left\{\lim _{n \rightarrow \infty} \tau^{+, n}=\infty\right\}=1$, if and only if

$$
\sum_{n \geq 0} \lambda_{n}^{-1}=\infty
$$

see Feller (1950, XVII.4). Examples of explosive processes can be found e.g. in Snyder and Miller (1991), see Example 6.3.1 with $\lambda_{n}=(n+1)^{2}$.

Let $\left\langle c_{n}, \lambda_{n}\right\rangle, n \geq 0$, be the sequence of states of the process $L=L(t)$.

We find an explicit formula for the density function $p=p(x, t)$ of $L(t)$ in the form

$$
p(x, t)=\sum_{n=0}^{\infty} p_{n}(x, t), \quad x \in(-\infty, \infty), t \geq 0 .
$$

Here $p_{n}(\cdot, t)$ are the density functions of $L(t) \mathbf{1}_{\{N(t)=n\}}$, which are determined by the set of $n+1$ states $\left\{\left\langle c_{k}, \lambda_{k}\right\rangle \mid k=0, \ldots, n\right\}$.

Note that if there are no switchings, $N(t)=0, L(t)=c_{0} t$, then the distribution of $L(t) \mathbf{1}_{\{N(t)=0\}}$ corresponds to Dirac's $\delta$-measure located at $c_{0} t$ (with probability 
$\left.\pi(t ; 0)=\mathrm{e}^{-\lambda_{0} t}\right)$, i. e., $p_{0}(x, t)=\mathrm{e}^{-\lambda_{0} t} \delta\left(x-c_{0} t\right)$. Moreover, functions $p_{n}$ follow the set of integral equations, $n \geq 1$

$$
p_{n}(x, t)=\lambda_{0} \int_{0}^{t} \mathrm{e}^{-\lambda_{0} u} \widetilde{p}_{n-1}\left(x-c_{0} u, t-u\right) \mathrm{d} u,
$$

where $\widetilde{p}_{n-1}$ are determined by the set parameters $\left\{\left\langle c_{k}, \lambda_{k}\right\rangle \mid k=1, \ldots, n\right\}$, beginning with $\left\langle c_{1}, \lambda_{1}\right\rangle$.

The detailed properties and the explicit formulae for the distributions of $L(t)$, $t \geq 0$, with the states $\left\langle c_{0}, \lambda_{0}\right\rangle$ and $\left\langle c_{1}, \lambda_{1}\right\rangle$, alternating at times $\tau^{+, n}, n \geq 0$, are known. The density functions $p_{n}(x, t)$ of $L(t) \mathbf{1}_{\{N(t)=n\}}, n \geq 1$, are given by

$$
\begin{aligned}
p_{n}(x, t)= & \frac{\lambda_{0}^{[(n+1) / 2]} \lambda_{1}^{[n / 2]}}{[n / 2] ![(n-1) / 2] !} \tau_{*}{ }^{[n / 2]}\left(t-\tau_{*}\right)^{[(n-1) / 2]} \\
& \times \frac{\exp \left(-\lambda_{0} \tau_{*}-\lambda_{1}\left(t-\tau_{*}\right)\right)}{c_{0}-c_{1}} \mathbf{1}_{\left\{0<\tau_{*}<t\right\}},
\end{aligned}
$$

where $\tau_{*}=\frac{x-c_{1} t}{c_{0}-c_{1}}, t-\tau_{*}=\frac{c_{0} t-x}{c_{0}-c_{1}},[\cdot]$ denotes the integer part and the initial state is $\left\langle c_{0}, \lambda_{0}\right\rangle$. See Kolesnik and Ratanov (2013, Chapter 4), where system (2.10) is solved for the case of alternating states.

This result can be also obtained by using the moment generating functions

$$
\psi_{n}(z, t)=\mathbb{E}\left\{\mathrm{e}^{z L(t)} \mathbf{1}_{\{N(t)=n\}}\right\}, \quad n \geq 1 .
$$

The explicit formulae for $\psi_{n}$ see in López and Ratanov (2014, Theorem 2.1).

In this paper we study the distribution of $L(t)$, when all states $\left\langle c_{n}, \lambda_{n}\right\rangle, n \geq 0$, are distinct. By Ratanov (2014, Theorem 3.1) in this case the moment generating functions are

$$
\psi_{n}(z, t)=\mathbb{E}\left\{\mathrm{e}^{z L(t)} \mathbf{1}_{\{N(t)=n\}}\right\}=\Lambda_{n} \sum_{k=0}^{n} \kappa_{n, k}(\tilde{\lambda}) \mathrm{e}^{-\tilde{\lambda}_{k} t}, \quad n \geq 1 .
$$

Here the linear functions $\tilde{\lambda}_{k}=\tilde{\lambda}_{k}(z)=\lambda_{k}-c_{k} z, 0 \leq k \leq n$, are distinct, and $|z|$ is sufficiently small, such that all $\tilde{\lambda}_{k}(z)$ remain to be positive, $\tilde{\lambda}_{k}(z)>0,0 \leq k \leq n$. The latter means that

$$
\text { if } c_{k}>0 \text {, then } z<\lambda_{k} / c_{k} ; \quad \text { if } c_{k}<0 \text {, then } z>\lambda_{k} / c_{k}, \quad 0 \leq k \leq n \text {. }
$$

If some of states are equal, formula (2.11) can be modified.

It is easy to see that

$$
\kappa_{n, k}(\tilde{\lambda})=\prod_{j=0, j \neq k}^{n}\left(\lambda_{j}-\lambda_{k}-z\left(c_{j}-c_{k}\right)\right)^{-1}=\kappa_{n, k}(\vec{c}, \vec{\lambda}) \cdot \prod_{\substack{j=0 \\ c_{j} \neq c_{k}}}^{n}\left(b_{j, k}-z\right)^{-1},
$$

where $\kappa_{n, k}(\vec{c}, \vec{\lambda})$ are defined by (2.1) and $b_{j, k}=\frac{\lambda_{j}-\lambda_{k}}{c_{j}-c_{k}}$, if $c_{j} \neq c_{k}$. Hence, by (2.11)

$$
\psi_{n}(z, t)=\Lambda_{n} \sum_{k=0}^{n} \kappa_{n, k}(\vec{c}, \vec{\lambda}) \mathrm{e}^{-\lambda_{k} t} \times \frac{\mathrm{e}^{z c_{k} t}}{\prod_{j=0, c_{j} \neq c_{k}}^{n}\left(b_{j, k}-z\right)}
$$

for $z$ satisfying condition $(*)$. The distribution of $L(t)$ can be obtained by inverse Laplace transformation of (2.12), i. e., one can find the density function $p_{n}$ 
satisfying the equation

$$
\int_{-\infty}^{\infty} \mathrm{e}^{z x} p_{n}(x, t) \mathrm{d} x=\psi_{n}(z, t)
$$

Throughout the paper we will use the notations:

$$
\begin{gathered}
B_{j, k}=-b_{j, k} c_{k}+\lambda_{k}=\frac{\lambda_{k} c_{j}-\lambda_{j} c_{k}}{c_{j}-c_{k}}, \quad \text { and } \quad \tau_{j, k}=\tau_{j, k}(x, t)=\frac{x-c_{k} t}{c_{j}-c_{k}}, \\
c_{j} \neq c_{k}, \quad j, k \geq 0 .
\end{gathered}
$$

Notice that $b_{j, k}=b_{k, j}, B_{j, k}=B_{k, j}, \tau_{j, k}(x, t)+\tau_{k, j}(x, t) \equiv t$ and

$$
\begin{gathered}
\lambda_{j} \tau_{j, k}(x, t)+\lambda_{k} \tau_{k, j}(x, t) \equiv b_{j, k} x+B_{j, k} t \equiv b_{j, k}\left(x-c_{k} t\right)+\lambda_{k} t, \\
c_{j} \neq c_{k}, j, k \geq 0 .
\end{gathered}
$$

We will consider two cases:

A1: all $b_{j, k}$ are distinct, if they are defined:

$$
b_{l, k} \neq b_{j, k}\left(\text { for all } l \neq j, c_{l} \neq c_{k}, c_{j} \neq c_{k}\right) \text {; }
$$

B1: all $b_{j, k}$ are identical, $b_{j, k}=\beta, 0 \leq j, k \leq n$ (for $c_{j} \neq c_{k}$ ).

By applying (2.3) one can notice that in case A1 the moment generating function $\psi_{n},(2.12)$, becomes

$$
\psi_{n}(z, t)=\Lambda_{n} \sum_{k=0}^{n} \kappa_{n, k}(\vec{c}, \vec{\lambda}) \mathrm{e}^{-\lambda_{k} t} \times \sum_{\substack{j=0 \\ c_{j} \neq c_{k}}}^{n} \kappa_{n, j}\left(\vec{b}_{k}\right) \frac{\mathrm{e}^{z c_{k} t}}{b_{j, k}-z} .
$$

Here $\kappa_{n, j}\left(\vec{b}_{k}\right)$ is defined similarly to $(2.2)$,

$$
\kappa_{n, j}\left(\vec{b}_{k}\right)=\prod_{\substack{l=0, l \neq j, c_{l} \neq c_{k}, c_{j} \neq c_{k}}}^{n}\left(b_{l, k}-b_{j, k}\right)^{-1}
$$

Note that in case B1 functions

$$
\lambda_{j} \tau_{j, k}(x, t)+\lambda_{k} \tau_{k, j}(x, t) \equiv \lambda_{k} t+\beta\left(x-c_{k} t\right), \quad j \in\{0, \ldots, n\},
$$

do not depend on $j$, see (2.14). Moreover, in this case by $(2.12) \psi_{n}(z, t)$ becomes

$$
\psi_{n}(z, t)=\Lambda_{n} \sum_{k=0}^{n} \kappa_{n, k}(\vec{c}, \vec{\lambda}) \mathrm{e}^{-\lambda_{k} t} \times \frac{\mathrm{e}^{z c_{k} t}}{(\beta-z)^{m}}
$$

The following theorem proposes some explicit formulae for the density functions $p_{n}=p_{n}(x, t)$ of $L(t) \mathbf{1}_{\{N(t)=n\}}, t>0, n \geq 1$.

\section{Theorem 2.1. • In case A1:}

$$
p_{n}(x, t)=\Lambda_{n} \sum_{\substack{k, j=0, c_{j} \neq c_{k}}}^{n} \phi_{c_{k} t, b_{j, k}}(x) \kappa_{n, k}(\vec{c}, \vec{\lambda}) \kappa_{n, j}\left(\vec{b}_{k}\right) \cdot \exp \left(-\lambda_{j} \tau_{j, k}(x, t)-\lambda_{k} \tau_{k, j}(x, t)\right) .
$$

- In case B1:

$$
p_{n}(x, t)=\Lambda_{n} \sum_{k=0}^{n} \phi_{c_{k} t, \beta}(x) \kappa_{n, k}(\vec{c}, \vec{\lambda}) \cdot \frac{\left(x-c_{k} t\right)^{m}}{m !} \exp \left(-\lambda_{k} t-\beta\left(x-c_{k} t\right)\right) \text {. }
$$

Here for each $k, k \in\{0, \ldots, n\}$, we denote by $m=m_{n, k}$ the number of $j$, $j \in\{0, \ldots, n\}$, such that $c_{j} \neq c_{k}$. 
Function $\phi_{a, b}(x)$ is defined in Appendix,

$$
\phi_{a, b}(x)=\left\{\begin{aligned}
\mathbf{1}_{\{x>a\}}, & \text { if } b>0, \\
-\mathbf{1}_{\{x<a\}}, & \text { if } b<0 .
\end{aligned}\right.
$$

Proof: In cases A1 and B1 we apply the inverse Laplace transform to functions $\psi_{n}(\cdot, t)$, defined by (2.15) and (2.17) respectively. Formulae (2.18)-(2.19) follow by Proposition 1 of Appendix.

If all $b_{j, k}$ are distinct, then by (2.15) and formula (1) of Appendix we obtain

$$
p_{n}(x, t)=\Lambda_{n} \sum_{\substack{k, j=0, c_{j} \neq c_{k}}}^{n} \kappa_{n, k}(\vec{c}, \vec{\lambda}) \mathrm{e}^{-\lambda_{k} t} \phi_{c_{k} t, b_{j, k}}(x) \kappa_{n, j}\left(\vec{b}_{k}\right) \exp \left(-b_{j, k}\left(x-c_{k} t\right)\right) .
$$

Moreover, $b_{j, k}\left(x-c_{k} t\right)+\lambda_{k} t=b_{j, k} x+B_{j, k} t \equiv \lambda_{j} \tau_{j k}(x, t)+\lambda_{k} \tau_{k j}(x, t)$.

Similarly, (2.19) follows from (2.17) and formula (1) of Appendix.

Remark 2.2. In a situation comprised between the extreme cases A1 and B1 similar formulae can also be obtained. This is the usual modifications based on partial fraction decomposition of $\psi_{n}$, which can be applied if some (not all) of $b_{j, k}$ are equal.

Remark 2.3. By definition (2.4) after $n$ steps the support of random variable $L(t)$ is compact. If $N(t)=n$, then

$$
L(t) \in\left[a_{n} t, A_{n} t\right], \quad \forall t, t>0, \quad n \geq 1 .
$$

Here $a_{n}=\min _{0 \leq k \leq n}\left\{c_{k}\right\}, \quad A_{n}=\max _{0 \leq k \leq n}\left\{c_{k}\right\}$.

By using representation (2.18) one can also show

$$
\mathbb{P}\left\{L(t) \in\left[a_{n} t, A_{n} t\right] \mid N(t)=n\right\}=1, \quad t>0, \quad n \geq 1,
$$

(in the case of distinct $b_{j, k}$ ). Indeed, it is easy to see that

$$
\kappa_{n, k}(\vec{c}, \vec{\lambda}) \kappa_{n, j}\left(\vec{b}_{k}\right)+\kappa_{n, j}(\vec{c}, \vec{\lambda}) \kappa_{n, k}\left(\vec{b}_{j}\right)=0, \quad j, k \in\{0, \ldots, n\}, j \neq k, n \geq 1 .
$$

Therefore, (2.18) can be transformed to

$$
p_{n}(x, t)=\Lambda_{n} \sum_{\substack{k, j=0 \\ k<j, c_{j} \neq c_{k}}}^{n}\left|\kappa_{n, k}(\vec{c}, \vec{\lambda}) \kappa_{n, j}\left(\vec{b}_{k}\right)\right| e_{j, k}(x, t),
$$

where

$$
e_{j, k}(x, t) \equiv e_{k, j}(x, t)=\exp \left(-\lambda_{j} \tau_{j, k}(x, t)-\lambda_{k} \tau_{k, j}(x, t)\right) \mathbf{1}_{\left\{x \text { is between } c_{j} t \text { and } c_{k} t\right\}} .
$$

This gives (2.21).

Further, by the definition we have

$$
\int_{-\infty}^{\infty} p_{n}(x, t) \mathrm{d} x=\pi(t ; n)=\mathbb{P}\{N(t)=n\}, \quad n \geq 1 .
$$


This also follows by integrating in (2.18) and (2.19). Indeed, if all $b_{j, k}$ are distinct, by (2.20) and (2.3) (with $\vec{b}_{k}$ instead of $\vec{c}$ ) one can obtain

$$
\begin{aligned}
\int_{-\infty}^{\infty} p_{n}(x, t) \mathrm{d} x & =\Lambda_{n} \sum_{k, j=0, c_{j} \neq c_{k}}^{n} \mathrm{e}^{-\lambda_{k} t} \kappa_{n, k}(\vec{c}, \vec{\lambda}) \kappa_{n, j}\left(\vec{b}_{k}\right) b_{j, k}^{-1} \\
& =\Lambda_{n} \sum_{k=0}^{n} \mathrm{e}^{-\lambda_{k} t} \kappa_{n, k}(\vec{c}, \vec{\lambda})\left[\prod_{j=0, c_{j} \neq c_{k}}^{n} b_{j, k}^{-1}\right]=\Lambda_{n} \sum_{k=0}^{n} \kappa_{n, k}(\vec{\lambda}) \mathrm{e}^{-\lambda_{k} t} \\
& =\pi(t ; n) .
\end{aligned}
$$

In the case of identical $b_{j, k}$ the same observations similarly follow from (2.19).

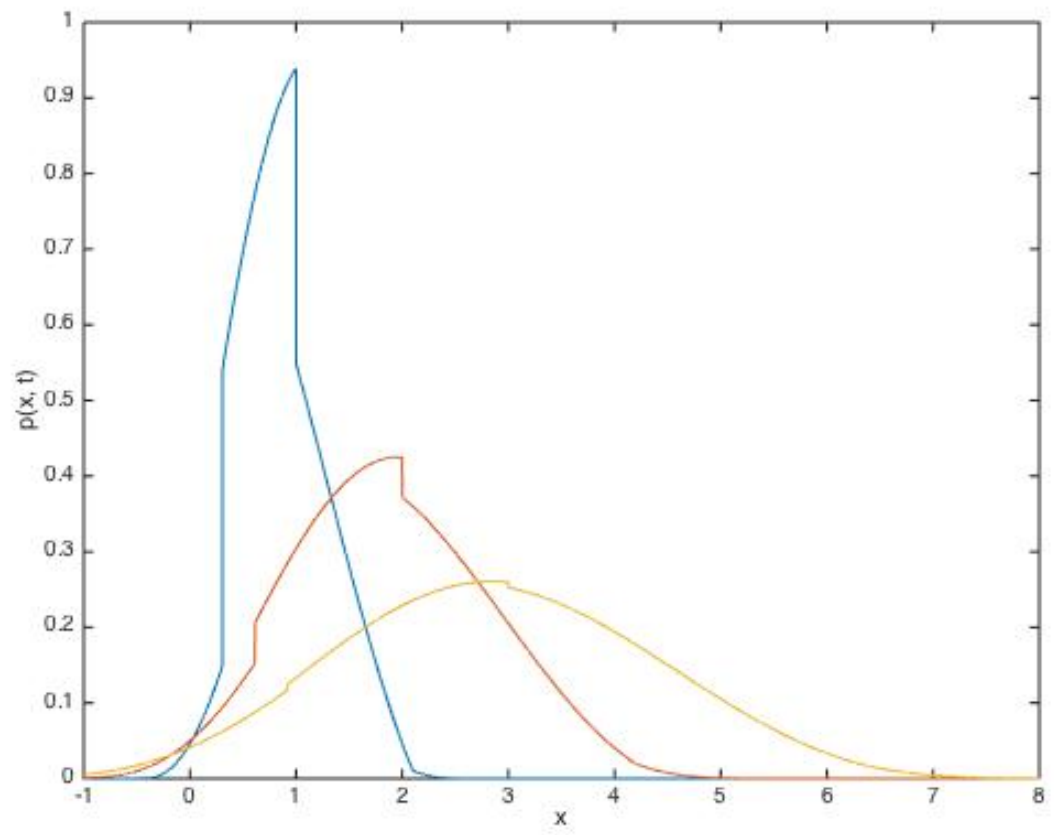

Figure 2.1. Density functions $p(x, t)$ with $c_{n}=1+(-1)^{n} \ln (n+1)$ and $\lambda_{n}=2$ and $t=1,2,3$ (from left to right).

Figure 2.1 displays the density functions in the case of slowly accelerating movement with reversals, $c_{n}=(-1)^{n} \ln (n+1)$ (the switching intensity is constant).

Next, we present some other examples.

Example 2.4. Consider an accelerating movement with linearly increasing velocities and switching intensities.

Let $c_{n}=c(n+1)$ and $\lambda_{n}=\lambda(n+1), n \geq 0$. 


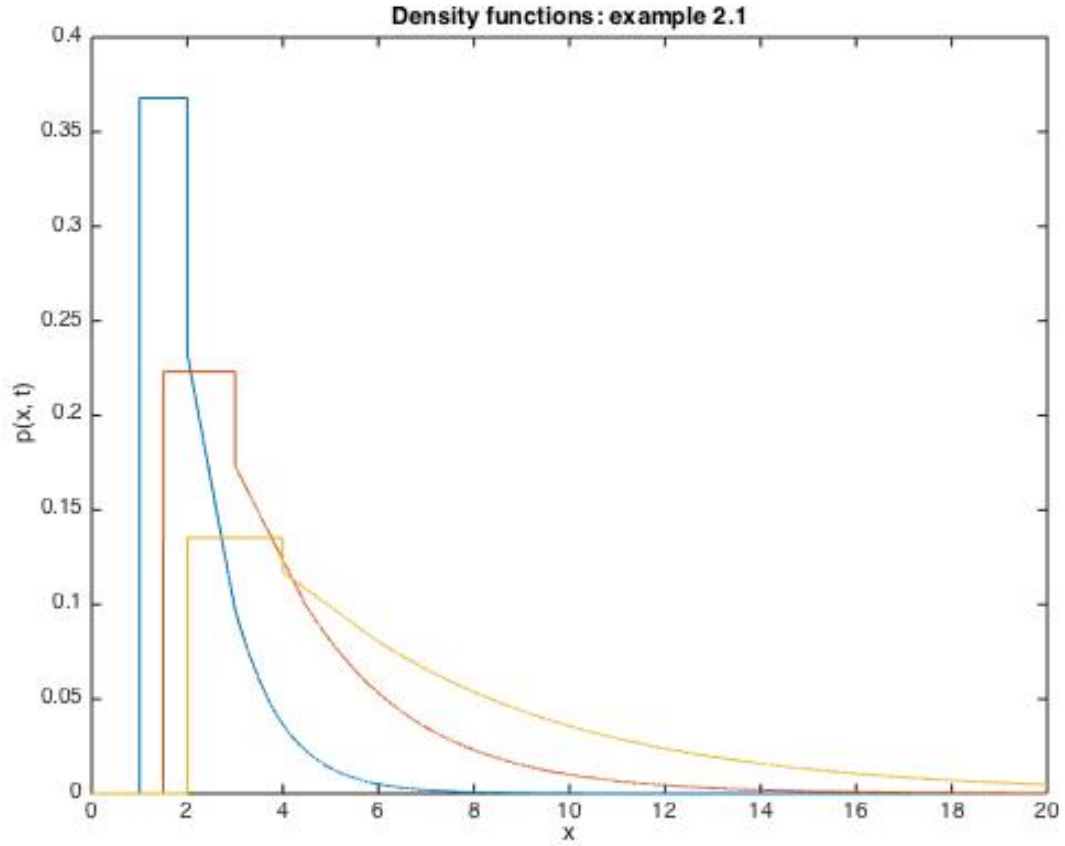

Figure 2.2. Density functions $p(x, t)$ with $c_{n}=n+1$ and $\lambda_{n}=$ $n+1$ and $t=1,1.5,2$ (from left to right).

In this case $b_{j, k} \equiv \lambda / c, B_{j, k} \equiv 0, \kappa_{n, k}(\vec{c})=\frac{(-1)^{k}}{c^{n} k !(n-k) !}, \Lambda_{n}=\lambda^{n} n !$ and by $(2.19)$

$$
p_{n}(x, t)=n(\lambda / c)^{n} \mathrm{e}^{-\lambda x / c} \sum_{k=0}^{n} \frac{(-1)^{k}\left(x-c_{k} t\right)^{n-1}}{k !(n-k) !} \mathbf{1}_{\left\{x>c_{k} t\right\}}, \quad n \geq 1 .
$$

Therefore, the absolutely continuous part of the distribution of $L(t)$ can be simplified by

$$
\begin{gathered}
\sum_{n=1}^{\infty} p_{n}(x, t)=\mathrm{e}^{-\lambda x / c} \sum_{n=1}^{\infty} n(\lambda / c)^{n} \sum_{k=0}^{n} \frac{(-1)^{k}\left(x-c_{k} t\right)^{n-1}}{k !(n-k) !} \mathbf{1}_{\left\{x>c_{k} t\right\}} \\
=\frac{\lambda}{c} \mathrm{e}^{-\lambda t} \mathbf{1}_{\{x>c t\}}+\mathrm{e}^{-\lambda x / c} \sum_{k=1}^{\infty} \frac{(-1)^{k}}{k !} \mathbf{1}_{\left\{x>c_{k} t\right\}}\left[\sum_{n=k}^{\infty} n \frac{(\lambda / c)^{n}}{(n-k) !}\left(x-c_{k} t\right)^{n-1}\right] \\
=\frac{\lambda}{c} \mathrm{e}^{-\lambda t} \mathbf{1}_{\{x>c t\}}+\frac{\lambda}{c} \mathrm{e}^{-\lambda x / c} \sum_{k=1}^{\infty} \frac{(-1)^{k}}{k !} \eta_{k}(x, t)^{k-1} \mathbf{1}_{\left\{x>c_{k} t\right\}} \sum_{n=0}^{\infty}(n+k) \frac{\eta_{k}(x, t)^{n}}{n !}
\end{gathered}
$$

where $\eta_{k}(x, t)=\lambda\left(x-c_{k} t\right) / c$. 
By summing up in the latter series one can obtain the density function $p=p(x, t)$ of $L(t)$

$$
p(x, t)=\mathrm{e}^{-\lambda t}\left[\delta(x-c t)+\frac{\lambda}{c} \sum_{k=0}^{\infty} \frac{(-1)^{k} \eta_{k}(x, t)^{k-1}}{k !}\left(k+\eta_{k}(x, t)\right) \mathrm{e}^{-k \lambda t} \mathbf{1}_{\left\{x>c_{k} t\right\}}\right] .
$$

Notice that for fixed $x$ and $t$ the sum in (2.22) is of finite number of terms. Figure 2.2 shows the absolutely continuous part of the density function $p(x, t)$ defined by (2.22).

Example 2.5. Consider an accelerating movement with linearly increasing velocities and constant intensities.

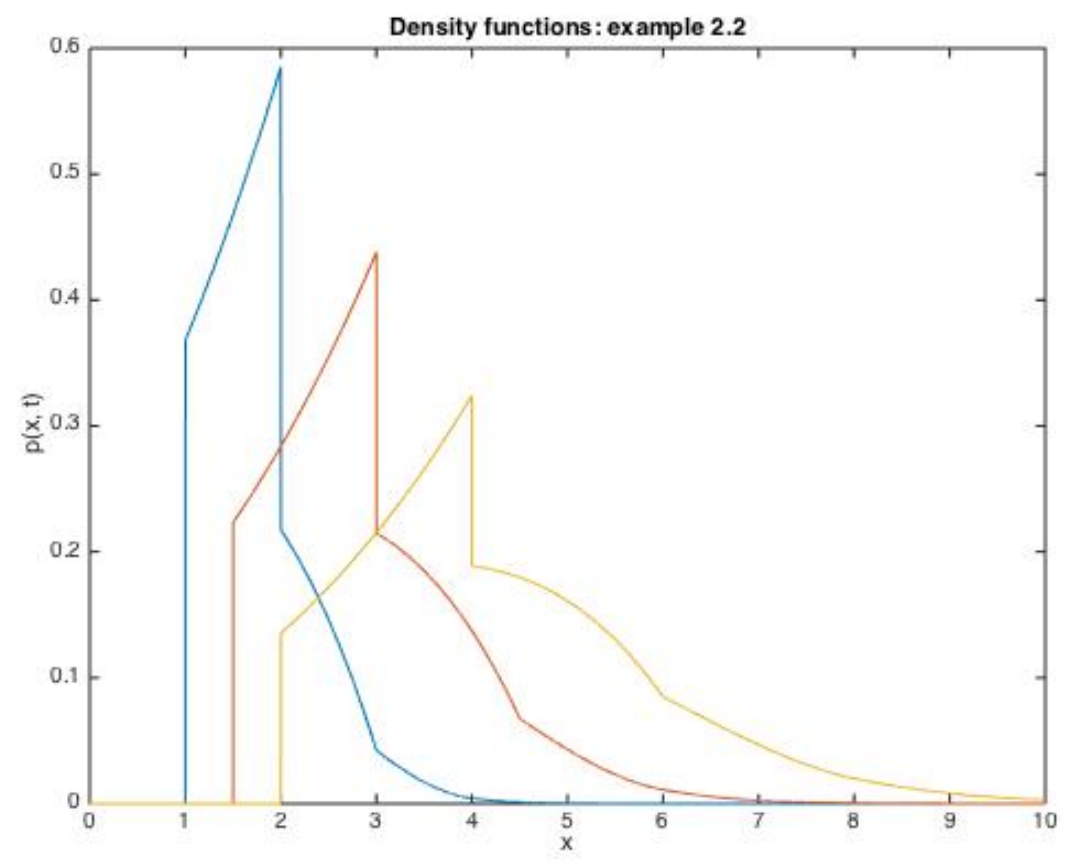

Figure 2.3. Density functions $p(x, t)$ with $c_{n}=n+1$ and $\lambda_{n}=1$ and $t=1,1.5,2$ (from left to right).

Let $c_{n}=c(1+n), c>0$, and $\lambda_{n} \equiv \lambda, n \geq 0$. In this case the density function $p=p(x, t)=\sum_{n=0}^{\infty} p_{n}(x, t)$ of $L(t), t>0$, can be simplified up to

$$
\begin{aligned}
p(x, t)=\mathrm{e}^{-\lambda t}\left[\delta\left(x-c_{0} t\right)\right. & \\
& \left.+\frac{\lambda}{c} \sum_{k=0}^{\infty} \frac{(-1)^{k} \eta_{k}(x, t)^{(k-1) / 2}}{k !} I_{k-1}\left(2 \sqrt{\eta_{k}(x, t)}\right) \mathbf{1}_{\left\{x>c_{k} t\right\}}\right],
\end{aligned}
$$

where $I_{\nu}=I_{\nu}(z)=\sum_{n=0}^{\infty} \frac{(z / 2)^{\nu+2 n}}{n ! \Gamma(\nu+n+1)}, \nu \geq 0 ; I_{-1}=I_{1}$ are modified Bessel functions; $\eta_{k}(x, t), k \geq 0$, are defined in Example 2.4. 
Indeed, by (2.19) the functions $p_{n}, n \geq 1$, become

$$
p_{n}(x, t)=\frac{(\lambda / c)^{n}}{(n-1) !} \mathrm{e}^{-\lambda t} \sum_{k=0}^{n} \frac{(-1)^{k}\left(x-c_{k} t\right)^{n-1}}{k !(n-k) !} \mathbf{1}_{\left\{x>c_{k} t\right\}} .
$$

Therefore,

$$
\begin{aligned}
p(x, t)= & \sum_{n=0}^{\infty} p_{n}(x, t) \\
= & \mathrm{e}^{-\lambda t}\left[\delta\left(x-c_{0} t\right)+\sum_{n=1}^{\infty} \frac{(\lambda / c)^{n}}{(n-1) !} \sum_{k=0}^{n} \frac{(-1)^{k}\left(x-c_{k} t\right)^{n-1}}{k !(n-k) !} \mathbf{1}_{\left\{x>c_{k} t\right\}}\right] \\
= & \mathrm{e}^{-\lambda t}\left[\delta\left(x-c_{0} t\right)+\sum_{n=1}^{\infty} \frac{(\lambda / c)^{n}}{(n-1) ! n !}(x-c t)^{n-1} \mathbf{1}_{\{x>c t\}}\right. \\
& \left.+\sum_{k=1}^{\infty} \frac{(-1)^{k}}{k !} \mathbf{1}_{\left\{x>c_{k} t\right\}} \sum_{n=k}^{\infty} \frac{\left(x-c_{k} t\right)^{n-1}}{(n-1) !(n-k) !}(\lambda / c)^{n}\right]
\end{aligned}
$$

which by definition of $I_{\nu}$ gives (2.23).

Notice that for fixed $x$ and $t$ the sum in (2.23) is of finite number of terms. Figure 2.3 represents the absolutely continuous part of the density functions expressed by (2.24).

Example 2.6. Let $L=L(t)$ be a process with alternating velocities $c_{2 n}=c, c_{2 n+1}=$ $-v, n \geq 0$, and with switching intensities, $\lambda_{2 n}=\lambda(1+n), \lambda_{2 n+1}=\mu(1+n)$, $\lambda, \mu>0$. This example has been analysed in detail by Di Crescenzo and Martinucci (2010).

Formulae (2.18) enable to repeat their result and, moreover, solve the unsolved PDEs, written at the beginning of Section 3 in Di Crescenzo and Martinucci (2010).

One can see that in this case the coefficients in (2.18) are given by

$$
\left\{\begin{aligned}
\Lambda_{2 n} & =\lambda^{n} \mu^{n}(n !)^{2}, \quad \Lambda_{2 n+1}=\lambda^{n+1} \mu^{n} n !(n+1) ! \\
\tau_{2 k, 2 j+1} & =\frac{x+v t}{c+v}, \quad \tau_{2 j+1,2 k}=t-\tau_{2 k, 2 j+1}=\frac{c t-x}{c+v}, \\
b_{2 j, 2 k+1} & =\frac{\lambda(1+j)-\mu(1+k)}{c+v}, \\
b_{2 j+1,2 k} & =\frac{\lambda(1+k)-\mu(1+j)}{c+v}, \\
\kappa_{2 n, 2 k}(\vec{c}, \vec{\lambda}) & =(-1)^{n+k}(c+v)^{-n} \lambda^{-n}[k !(n-k) !]^{-1}, \\
\kappa_{2 n, 2 k+1}(\vec{c}, \vec{\lambda}) & =(-1)^{k}(c+v)^{-n-1} \mu^{-n+1}[k !(n-k-1) !]^{-1}, \\
\kappa_{2 n+1,2 k}(\vec{c}, \vec{\lambda}) & =(-1)^{n+k}(c+v)^{-n-1} \lambda^{-n}[k !(n-k) !]^{-1}, \\
\kappa_{2 n+1,2 k+1}(\vec{c}, \vec{\lambda}) & =(-1)^{k}(c+v)^{-n-1} \mu^{-n}[k !(n-k) !]^{-1}, \\
\kappa_{2 n, 2 j+1}\left(\vec{b}_{2 k}\right) & =(-1)^{n-j-1}(c+v)^{n-1} \mu^{-n+1}[j !(n-j-1) !]^{-1}, \\
\kappa_{2 n+1,2 j+1}\left(\vec{b}_{2 k}\right) & =(-1)^{n-j}(c+v)^{n} \mu^{-n}[j !(n-j) !]^{-1}, \\
\kappa_{2 n, 2 j}\left(\vec{b}_{2 k+1}\right) & =\kappa_{2 n+1,2 j}\left(\vec{b}_{2 k+1}\right)=(-1)^{j}(c+v)^{n} \lambda^{-n}[j !(n-j) !]^{-1},
\end{aligned}\right.
$$

for $n, k, j \in\{0, \ldots, n\}$. 
By summing up in (2.18) with (2.25) we get, for $-v t<x<c t$

$$
\begin{aligned}
& p_{2 n}(x, t)=\frac{n \mu}{c+v} \mathrm{e}^{-\lambda \tau_{*}-\mu\left(t-\tau_{*}\right)}\left(1-\mathrm{e}^{-\lambda \tau_{*}}\right)^{n}\left(1-\mathrm{e}^{-\mu\left(t-\tau_{*}\right)}\right)^{n-1}, \quad n \geq 1, \\
& p_{2 n+1}(x, t)=\frac{(n+1) \lambda}{c+v} \mathrm{e}^{-\lambda \tau_{*}-\mu\left(t-\tau_{*}\right)}\left(1-\mathrm{e}^{-\lambda \tau_{*}}\right)^{n}\left(1-\mathrm{e}^{-\mu\left(t-\tau_{*}\right)}\right)^{n}, \quad n \geq 0 .
\end{aligned}
$$

Here $\tau_{*}=\frac{x+v t}{c+v}$.

Furthermore, functions $p_{2 n}=f_{n}(x, t \mid c)$ and $p_{2 n+1}=b_{n}(x, t \mid c)$ defined by (2.26)-(2.27) solve PDEs of Di Crescenzo and Martinucci (2010, p.88). Functions $f_{n}(x, t \mid-v)$ and $b_{n}(x, t \mid-v)$ can be written similarly.

Formulae Di Crescenzo and Martinucci (2010, (3.5)-(3.6)) for the density functions $f(x, t \mid c)=\sum_{n=1}^{\infty} p_{2 n}(x, t)$ and $b(x, t \mid c)=\sum_{n=0}^{\infty} p_{2 n+1}(x, t)$ easily follow from (2.26) and (2.27). Multiple plots depicting the density functions $p=p(\cdot, t)$ with different $\lambda, \mu$ and $t$ are as well presented by Di Crescenzo and Martinucci (2010).

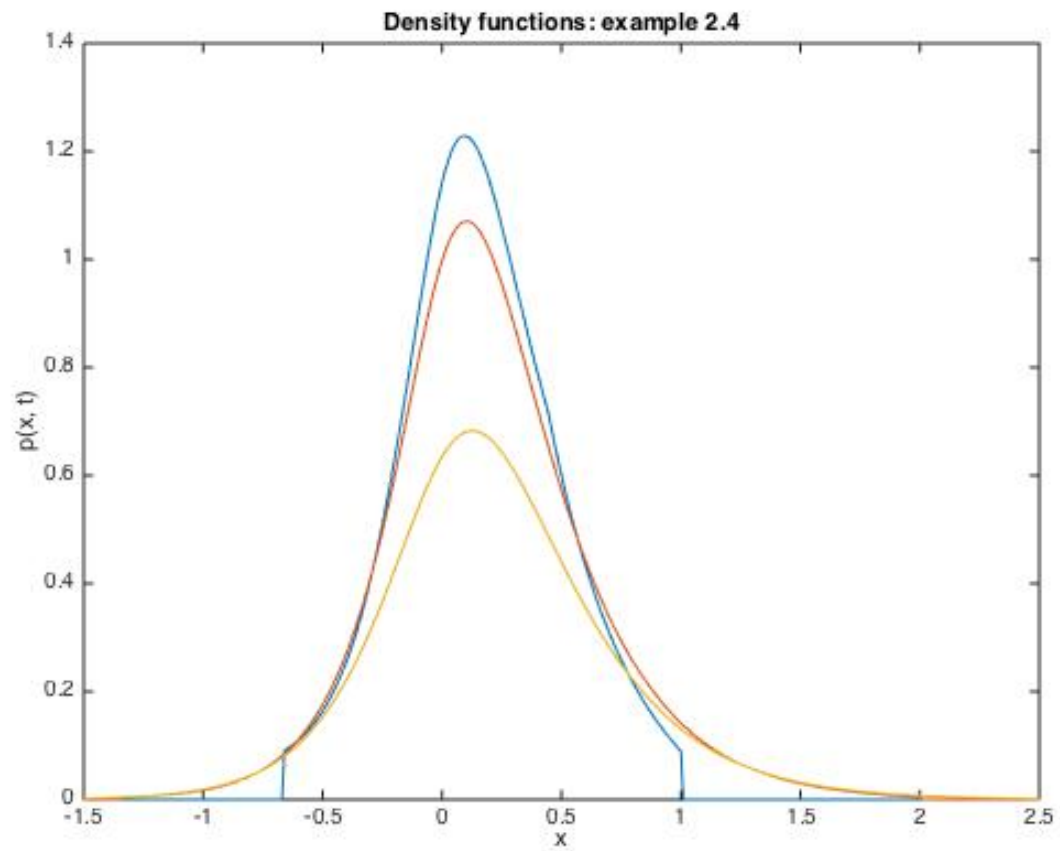

Figure 2.4. Density functions $p(x, t)$ with $c_{n}=(-2 / 3)^{n}$ and $\lambda_{n}=3$ and $t=1,2,3$ (from top to bottom).

Example 2.7. Consider the fading case, $c_{n}=(-a)^{n}, 0<a<1$, and $\lambda_{n} \equiv \lambda$, $\lambda>0, n \geq 0$. By (2.19) we have

$$
p_{n}(x, t)=\frac{\lambda^{n} \mathrm{e}^{-\lambda t}}{(n-1) !} \sum_{k=0}^{n} \kappa_{n, k}(\vec{c})\left(x-c_{k} t\right)^{n-1} \mathbf{1}\left\{x>c_{k} t\right\}, \quad n \geq 1
$$


In this case by "three-series theorem" $L(t)$ converges a. s., as $t \rightarrow \infty$. The distribution of $L(\infty)$ is given by

$$
\mathbb{P}\{L(\infty)<x\}=\int_{0}^{\infty}\left[1-F\left(\frac{y-x}{a}\right)\right] \mathrm{d} F(y),
$$

where

$$
F(x)=\left[1-s^{-1} \sum_{n=0}^{\infty}(-1)^{n} A_{n} \mathrm{e}^{-\lambda x / a^{2 n}}\right] \mathbf{1}_{\{x \geq 0\}}, \quad s=\sum_{n=0}^{\infty}(-1)^{n} A_{n} .
$$

Here $A_{n}=\frac{a^{n(n+1)}}{\left(1-a^{2}\right)^{n(n+1) / 2}}$, cf Samoilenko (2002).

Figure 2.4 displays the absolutely continuous part of the density functions of the fading movement, defined by (2.28).

2.2. First passage time. Let $x, x>0$, and $T=T(x)$ be the first passage time

$$
T(x)=\inf \{t \geq 0 \mid L(t)=x\} .
$$

In the case of alternating parameters, $c_{n} \in\left\{c_{0}, c_{1}\right\}, \lambda_{n} \in\left\{\lambda_{0}, \lambda_{1}\right\}, c_{0}>0>$ $c_{1}$, the distribution of $T(x)$ is well-studied. See Foong (1992), Foong and Kanno (1994), Orsingher (1995) and Pinsky (1991) for symmetric processes, $\left(\lambda_{0}=\lambda_{1}\right.$ and $c_{0}=-c_{1}$ ), Stadje and Zacks (2004) and López and Ratanov (2014, Theorem 3.1 ) in the asymmetric case. The case of alternating velocities $c,-v$ with linearly increasing reversal rates is studied by Di Crescenzo and Martinucci (2010).

Here we study the distribution of $T(x)$ assuming all velocities $c_{n}$ to be positive.

Under the non explosion condition, see (2.9),

$$
\sum_{n=0}^{\infty} \frac{c_{n}}{\lambda_{n}}=\infty
$$

we have $T(x)<\infty$, a.s. Notice that $c_{n} \tau_{n}$ is exponentially distributed, $\operatorname{Exp}\left(\lambda_{n} / c_{n}\right)$, $n \geq 0$.

The distribution of $T(x)$ possesses an atom at $x / c_{0}$,

$$
\mathbb{P}\left\{T(x)=x / c_{0}\right\}=\mathrm{e}^{-\lambda_{0} x / c_{0}} .
$$

We compute the absolutely continuous part of the distribution in the following two cases:

A2: all $B_{j, k}$ are distinct, if they are defined:

$$
B_{l, k} \neq B_{j, k}\left(\text { for all } l \neq j, c_{l} \neq c_{k}, c_{j} \neq c_{k}\right)
$$

B2: all $B_{j, k}$ are identical, $B_{j, k}=B, 0 \leq j, k \leq n$ (for $c_{j} \neq c_{k}$ ).

Here $B_{j, k}$ are defined by (2.13).

It is easy to see, that in the case $\mathrm{B} 2$ all $b_{j, k}$ are also equal: $b_{j, k} \equiv \beta$.

Let $g_{n}(t ; x)$ be the density function of the random variable $T(x) \mathbf{1}_{\{N(T(x))=n\}}$, $n \geq 1$.

Theorem 2.8. Let $c_{n}>0 \forall n$, and condition (2.30) be hold. The density functions $g_{n}(t ; x)$ are given by

- in case A2:

$$
g_{n}(t ; x)=-\Lambda_{n} c_{n} \sum_{\substack{k, j=0 \\ c_{j} \neq c_{k}}}^{n} c_{k}^{m-1} \kappa_{n, k}(\vec{c}, \vec{\lambda}) \kappa_{n, j}\left(-\vec{B}_{k}\right) \mathrm{e}^{-\left(B_{j, k} t+b_{j, k} x\right)} \mathbf{1}_{\left\{c_{k} t>x\right\}},
$$


where coefficients $\kappa_{n, j}\left(\vec{B}_{k}\right)$ are defined by (2.16);

- in case $\mathrm{B} 2$ :

$$
g_{n}(t ; x)=-\Lambda_{n} c_{n} \mathrm{e}^{-(B t+\beta x)} \sum_{k=0}^{n} \kappa_{n, k}(\vec{c}, \vec{\lambda}) \frac{\left(x-c_{k} t\right)^{m-1}}{(m-1) !} \mathbf{1}_{\left\{c_{k} t>x\right\}} .
$$

Here, as in Theorem 2.1, for each $k, k \in\{0, \ldots, n\}$, we denote by $m=m_{n, k}$ the number of $j, j \in\{0, \ldots, n\}$, such that $c_{j} \neq c_{k}$.

Proof: Let $N(T(x))=n, n \geq 1$. Under this condition we have

$$
\tau^{+, n} \leq T(x)<\tau^{+, n+1}, \quad n \geq 1,
$$

which is equivalent to

$$
\sum_{k=0}^{n-1} c_{k} \tau_{k} \leq x, \quad c_{n} \tau_{n}>x-\sum_{k=0}^{n-1} c_{k} \tau_{k},
$$

where $\tau_{k}$ are independent exponentially distributed, $\tau_{k} \sim \operatorname{Exp}\left(\lambda_{k}\right)$, random variables.

In this case by definition (2.4) we have

$$
x=L(T(x))=\sum_{k=0}^{n-1}\left(c_{k}-c_{n}\right) \tau_{k}+c_{n} T(x) .
$$

This equation gives

$$
T(x)=\frac{x-\sum_{k=0}^{n-1}\left(c_{k}-c_{n}\right) \tau_{k}}{c_{n}}=\frac{x}{c_{n}}-\sum_{k=0}^{n-1}\left(\frac{1}{c_{n}}-\frac{1}{c_{k}}\right) \xi_{k},
$$

where $\xi_{k}=c_{k} \tau_{k} \sim \operatorname{Exp}\left(\lambda_{k} / c_{k}\right), k \geq 0$, are independent.

Therefore by (2.33) we have

$$
\begin{aligned}
& \mathbb{E}\left[\mathrm{e}^{z T(x)} \mathbf{1}_{\{N(T(x))=n\}}\right] \\
& =\exp \left\{z x / c_{n}\right\} \mathbb{E}\left[\exp \left\{-z \sum_{k=0}^{n-1}\left(\frac{1}{c_{n}}-\frac{1}{c_{k}}\right) \xi_{k}\right\} \mathbf{1}_{\left\{\xi^{+, n} \leq x, \xi_{n}>x-\xi^{+, n}\right\}}\right] .
\end{aligned}
$$

Here $\xi^{+, n}=\sum_{k=0}^{n-1} \xi_{k}$.

Applying formula Ratanov $(2014,(2.5))$ for the joint distribution of $\left(\xi_{0}, \ldots, \xi_{n-1}\right)$ we obtain

$$
\begin{gathered}
\mathbb{E}\left[\mathrm{e}^{z T(x)} \mathbf{1}_{\{N(T(x))=n\}}\right] \\
=\Lambda_{n} C_{n}^{-1} \mathrm{e}^{-\left(\lambda_{n}-z\right) x / c_{n}} \int_{\Pi_{n}(x)} \exp \left\{-\sum_{k=0}^{n-1} y_{k}\left(z\left(\frac{1}{c_{n}}-\frac{1}{c_{k}}\right)+\left(\frac{\lambda_{k}}{c_{k}}-\frac{\lambda_{n}}{c_{n}}\right)\right)\right\} \mathrm{d} \vec{y} \\
=\Lambda_{n} C_{n}^{-1} \exp \left\{-\alpha_{n}(z) x\right\} \int_{\Pi_{n}(x)} \exp \left\{-\sum_{k=0}^{n-1} y_{k}\left(\alpha_{k}(z)-\alpha_{n}(z)\right)\right\} \mathrm{d} \vec{y}, \quad n \geq 1,
\end{gathered}
$$




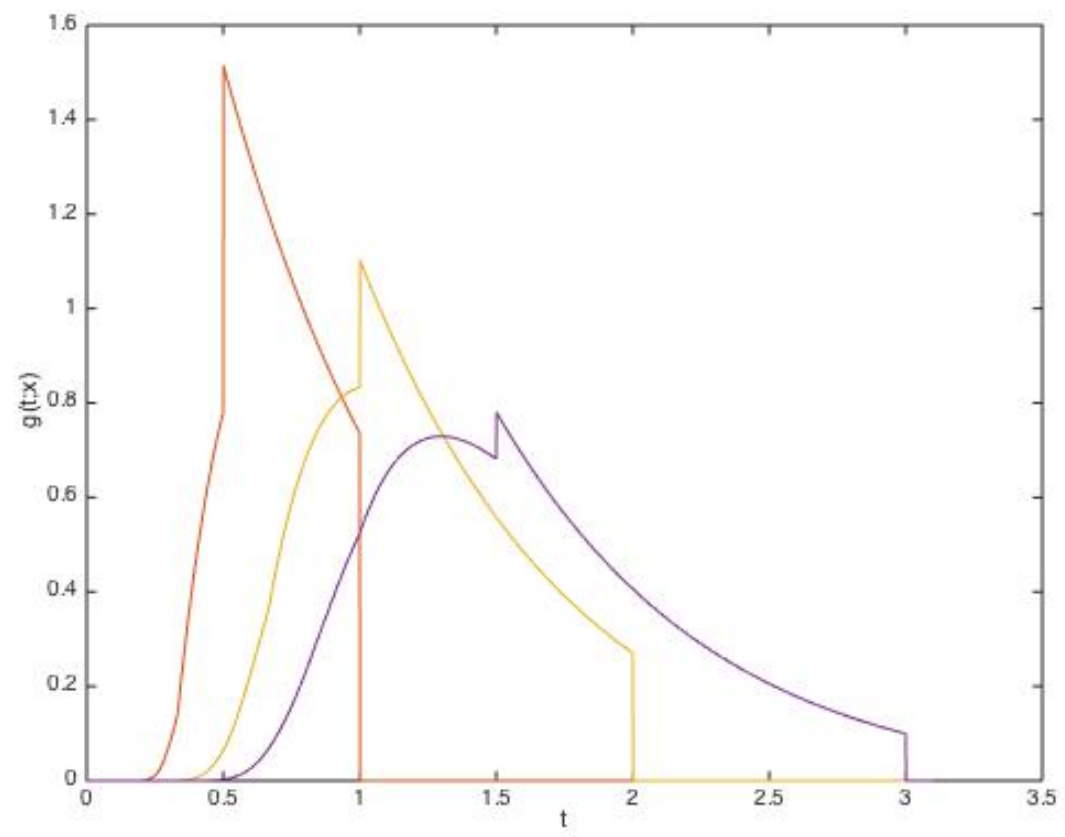

Figure 2.5. Density functions $g(t ; x)$ with $c_{n}=n+1$ and $\lambda_{n}=$ $n+1, n \geq 0$, and $x=1,2,3$ (from left to right).

where $C_{n}=\prod_{k=0}^{n-1} c_{k}, \alpha_{n}=\alpha_{n}(z)=\frac{\lambda_{n}-z}{c_{n}}, z<\lambda_{n}, \forall n$, and

$$
\Pi_{n}(x)=\left\{\vec{y}=\left(y_{0}, \ldots, y_{n-1}\right) \mid y^{(+, n)}=\sum_{i=0}^{n-1} y_{i}<x\right\} .
$$

By integrating in (2.34), see Proposition 3 (Appendix), we get

$$
\int_{0}^{\infty} \mathrm{e}^{z t} g_{n}(t ; x) \mathrm{d} t=\mathbb{E}\left[\mathrm{e}^{z T(x)} \mathbf{1}_{\{N(T(x))=n\}}\right]=\Lambda_{n} C_{n}^{-1} \sum_{k=0}^{n} \kappa_{n, k}(\vec{\alpha}(z)) \mathrm{e}^{-x \alpha_{k}(z)} .
$$

Note that

$$
\alpha_{j}-\alpha_{k}=-\frac{\lambda_{k} c_{j}-\lambda_{j} c_{k}}{c_{j} c_{k}}+\frac{c_{j}-c_{k}}{c_{j} c_{k}} z=\frac{c_{j}-c_{k}}{c_{j} c_{k}}\left(-B_{j k}+z\right) .
$$

Since

$$
\begin{aligned}
\kappa_{n, k}(\vec{\alpha}) & =\prod_{\substack{j=0 \\
c_{j} \neq c_{k}}}^{n}\left(z-B_{j, k}\right)^{-1} \times \prod_{\substack{j=0 \\
c_{j} \neq c_{k}}}^{n}\left[\left(c_{j}-c_{k}\right)^{-1} c_{j} c_{k}\right] \times \prod_{\substack{j=0 \\
c_{j}=c_{k}, j \neq k}}^{n}\left[\left(\lambda_{j}-\lambda_{k}\right)^{-1} c_{j}\right] \\
& =C_{n+1} c_{k}^{m_{n, k}-1} \kappa_{n, k}(\vec{c}, \vec{\lambda}) \prod_{\substack{j=0 \\
c_{j} \neq c_{k}}}^{n}\left(-B_{j, k}+z\right)^{-1}, \quad n \geq 1, \quad 0 \leq k \leq n,
\end{aligned}
$$




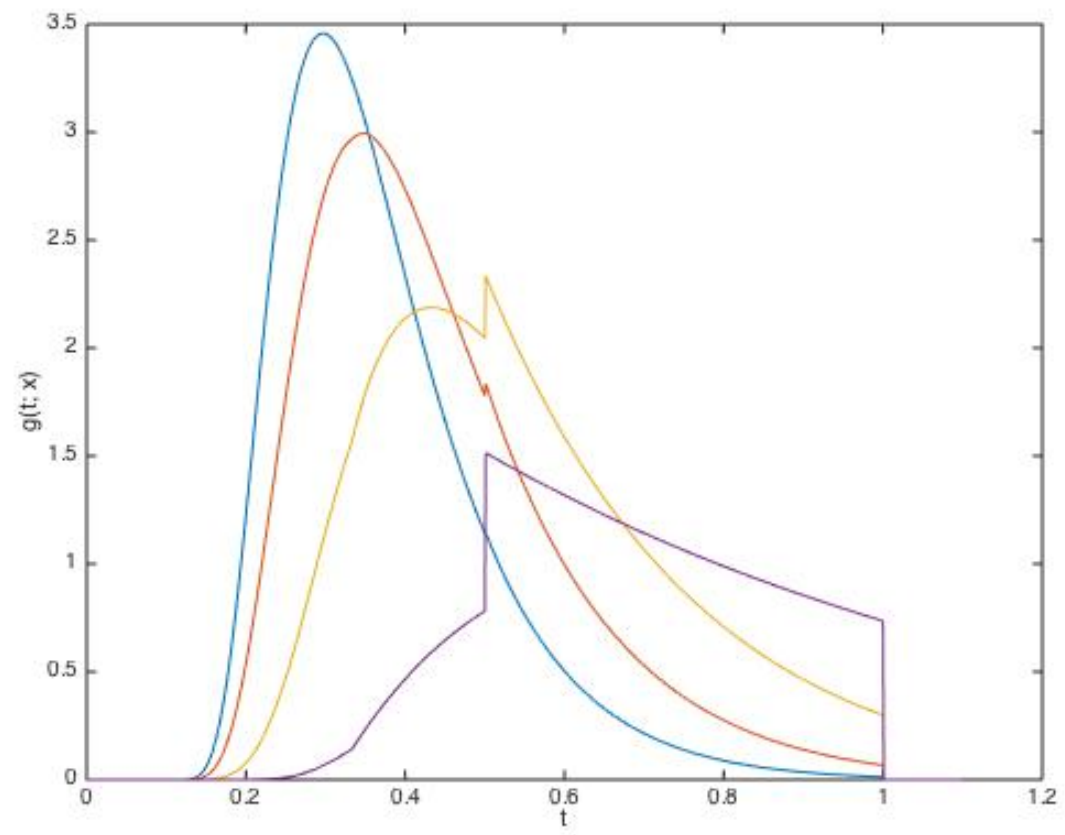

FIgURE 2.6. Density functions $g(t ; x)$ with $c_{n}=n+1$ and $\lambda_{n}=$ $\lambda(n+1), n \geq 0, x=1$ and $\lambda=7,5,3,1$ (from left to right).

we get

$$
\mathbb{E}\left[\mathrm{e}^{z T(x)} \mathbf{1}_{\{N(T(x))=n\}}\right]=\Lambda_{n} c_{n} \sum_{k=0}^{n} c_{k}^{m_{n, k}-1} \kappa_{n, k}(\vec{c}, \vec{\lambda}) \mathrm{e}^{-\lambda_{k} x / c_{k}} \frac{\mathrm{e}^{z x / c_{k}}}{\prod_{\substack{j=0 \\ c_{j} \neq c_{k}}}^{n}\left(-B_{j, k}+z\right)} .
$$

Here and below $m=m_{n, k}$ are defined as above, see Theorem 2.1.

If all $B_{j, k}$ are distinct (case A2), this expression can be simplified as (2.15):

$$
\begin{aligned}
& \mathbb{E}\left[\mathrm{e}^{z T(x)} \mathbf{1}_{\{N(T(x))=n\}}\right] \\
& =\Lambda_{n} c_{n} \sum_{k=0}^{n} c_{k}^{m-1} \kappa_{n, k}(\vec{c}, \vec{\lambda}) \mathrm{e}^{-\lambda_{k} x / c_{k}} \times \sum_{\substack{j=0 \\
c_{j} \neq c_{k}}}^{n} \kappa_{n, j}\left(-\vec{B}_{k}\right) \frac{\mathrm{e}^{z x / c_{k}}}{\left(-B_{j, k}+z\right)} .
\end{aligned}
$$

In case B2 we have

$$
\mathbb{E}\left[\mathrm{e}^{z T(x)} \mathbf{1}_{\{N(T(x))=n\}}\right]=\Lambda_{n} c_{n} \sum_{k=0}^{n} c_{k}^{m-1} \kappa_{n, k}(\vec{c}, \vec{\lambda}) \mathrm{e}^{-\lambda_{k} x / c_{k}} \frac{\mathrm{e}^{z x / c_{k}}}{(-B+z)^{m}}
$$

By applying the inverse Laplace transform, formulae (2.31)-(2.32) follow from Proposition 2 (see Appendix) and from the identity $B_{j, k}=-b_{j, k} c_{k}+\lambda_{k}, j \neq k$ (see (2.13)). 
The absolutely continuous part of the distribution of $T(x)$ is given by

$$
g(t ; x)=\sum_{n=1}^{\infty} g_{n}(t ; x)
$$

In the case of Example 2.4 the plots of $g(t ; x)$ are presented in Fig. 2.5 and Fig. 2.6 .

One can easily write the distribution of number of switchings till the first hitting of level $x$.

Corollary 2.9. Let $x>0$, all $c_{n}$ be positive, $c_{n}>0, n \geq 0$, and $T=T(x)$ be the first crossing time of the level $x,(2.29)$.

- If $\lambda_{0} / c_{0}, \lambda_{1} / c_{1}, \ldots, \lambda_{n} / c_{n}$ are distinct, then

$$
\mathbb{P}\{N(T)=n\}=\Lambda_{n} / C_{n} \sum_{k=0}^{n-1} \kappa_{n, k}(\lambda \overrightarrow{/ c}) \mathrm{e}^{-\lambda_{k} x / c_{k}} .
$$

- If $\lambda_{k} / c_{k}=a, k \in\{0, \ldots, n\}$, then

$$
\mathbb{P}\{N(T)=n\}=\frac{(a x)^{n}}{n !} \mathrm{e}^{-a x} .
$$

Proof: By (2.34) one can get

$$
\mathbb{P}\{N(T)=n\}=\frac{\Lambda_{n}}{C_{n}} \mathrm{e}^{-\lambda_{n} x / c_{n}} \int_{\Pi_{n}(x)} \exp \left(-\sum_{k=0}^{n-1} y_{k}\left(\frac{\lambda_{k}}{c_{k}}-\frac{\lambda_{n}}{c_{n}}\right)\right) \mathrm{d} \vec{y} .
$$

Formula (2.35) follows by integrating, see Proposition A3 (Appendix).

If all $\lambda_{k} / c_{k}=a$ are equal, then

$$
\mathbb{P}\{N(T)=n\}=a^{n} \mathrm{e}^{-a x} V\left|\Pi_{n}(x)\right|,
$$

which gives (2.36).

2.3. Jumps are added. Let $\left\{r_{n}\right\}_{n \geq 0}$ be the set of independent random variables which are independent of the driving Poisson process $N$. Let

$$
r(t)=\int_{0}^{t} r_{N(u-)} \mathrm{d} N(u)=\sum_{n=0}^{N(t)-1} r_{n}, \quad t>0 .
$$

be the compound Poisson process accompanying $L=L(t)$ : jumps occur at times of the velocity's switchings.

Process $L(t)+r(t), t \geq 0$, generalises well-known jump-telegraph processes. For the case of the alternating deterministic parameters $c_{n} \in\left\{c_{0}, c_{1}\right\}, r_{n} \in\left\{h_{0}, h_{1}\right\}$ process $L(t)+r(t), t \geq 0$, is studied by Kolesnik and Ratanov (2013, Chapter 4).

In general, the distribution of $[L(t)+r(t)] \cdot \mathbf{1}_{\{N(t)=n\}}$ is given by the density function

$$
q_{n}(x, t):=\int_{-\infty}^{\infty} p_{n}(x-y, t) \eta^{*, n}(\mathrm{~d} y)
$$

which is the convolution of the density $p_{n}(x, t)$ of $L(t)$ (see (2.18)-(2.19)) and the $n$-fold convolution of jumps' distributions $\eta_{k}(\mathrm{~d} x), 0 \leq k \leq n-1$. The density function $\phi^{L+r}(x, t)$ of $L(t)+r(t), t>0$, is given by

$$
\phi^{L+r}(x, t)=\mathrm{e}^{-\lambda_{0} t} \delta\left(x-c_{0} t\right)+\sum_{n=1}^{\infty} q_{n}(x, t)
$$


if the series converges.

Denote by $\overline{r_{n}}$ the expected jump amplitude, $\overline{r_{n}}=\int_{-\infty}^{\infty} x \eta_{n}(\mathrm{~d} x), n \geq 0$, and let $\rho_{n}=\sum_{k=0}^{n-1} \overline{r_{k}}, n \geq 1$.

Assume that the series uniformly converges:

$$
\alpha(t):=\sum_{n=0}^{\infty}\left(c_{n}+\lambda_{n} \overline{r_{n}}\right) \pi(t ; n)<\infty, \quad t \in[0, T],
$$

where $\pi(t ; n)=\mathbb{P}\{N(t)=n\}$. Moreover, let

$$
\left(c_{n}-\lambda_{n} \rho_{n}\right) \pi(t ; n) \rightrightarrows 0, \quad t \in[0, T]
$$

as $n \rightarrow \infty$.

Theorem 2.10. Under conditions (2.39)-(2.40) the expectation of $L(t)+r(t), t \geq$ 0 , is given by

$$
\mathbb{E}[L(t)+r(t)]=\int_{0}^{t} \alpha(u) \mathrm{d} u .
$$

Proof: By definitions (2.4), (2.37) and equations (2.5), (2.6) we have

$$
\begin{aligned}
& \frac{\mathrm{d}}{\mathrm{d} t} \mathbb{E}[L(t)+r(t)] \\
& =c_{0} \pi(t ; 0)+\lim _{N \rightarrow \infty} \sum_{n=1}^{N}\left(c_{n} \pi(t ; n)+\rho_{n}\left[-\lambda_{n} \pi(t ; n)+\lambda_{n-1} \pi(t ; n-1)\right]\right) \\
& =\lim _{N \rightarrow \infty}\left[\sum_{n=0}^{N-1}\left(c_{n}+\lambda_{n} \overline{r_{n}}\right) \pi(t ; n)+\left(c_{N}-\lambda_{N} \rho_{N}\right) \pi(t ; N)\right], \quad t \in[0, T],
\end{aligned}
$$

where the limit is uniform in $t \in[0, T]$. Therefore,

$$
\frac{\mathrm{d}}{\mathrm{d} t} \mathbb{E}[L(t)+r(t)]=\sum_{n=0}^{\infty}\left(c_{n}+\lambda_{n} \overline{r_{n}}\right) \pi(t ; n)=\alpha(t) .
$$

Equation (2.41) follows from (2.42).

Corollary 2.11. Let conditions (2.39)-(2.40) hold.

- If all $\lambda$ are distinct, $\lambda_{k} \neq \lambda_{n}, k \neq n$, then

$$
\mathbb{E}[L(t)+r(t)]=\sum_{n=0}^{\infty}\left(c_{n}+\lambda_{n} \overline{r_{n}}\right) \Lambda_{n} \sum_{k=0}^{n} \kappa_{n, k}(\vec{\lambda}) \lambda_{k}^{-1}\left(1-\mathrm{e}^{-\lambda_{k} t}\right) .
$$

- If $\lambda_{n} \equiv \lambda, n \geq 0$, then (by using the incomplete gamma-function $\gamma$ )

$$
\begin{aligned}
\mathbb{E}[L(t)+r(t)] & =\sum_{n=0}^{\infty}\left(c_{n} / \lambda+\overline{r_{n}}\right) \gamma(n+1, \lambda t) \\
& =\sum_{n=0}^{\infty}\left(c_{n} / \lambda+\overline{r_{n}}\right)\left[1-\mathrm{e}^{-\lambda t} \sum_{k=0}^{n} \frac{(\lambda t)^{k}}{k !}\right] .
\end{aligned}
$$

Proof: Equations (2.43)-(2.44) follows from (2.41) by integration of $\alpha(t)$. For (2.43) one can use (2.8), equation (2.44) follows by

$$
\alpha(t)=\sum_{n=0}^{\infty}\left(c_{n}+\lambda_{n} \overline{r_{n}}\right) \frac{(\lambda t)^{n}}{n !} \exp (-\lambda t), \quad t>0 .
$$


Remark 2.12. Formulae (2.41)-(2.44) seem more simple, than formula Ratanov (2014, (3.8)), which has been derived by differentiation of the corresponding moment generating function.

Corollary 2.13. Let

$$
c_{n}+\lambda_{n} \overline{r_{n}}=0, \quad \forall n \geq 0 .
$$

The process with jumps, $L(t)+r(t), t \geq 0$, is the martingale.

Proof: If (2.45) holds, then (see (2.41) and (2.39)) the expectation is zero,

$$
\mathbb{E}[L(t)+r(t)] \equiv 0
$$

The proof follows from renewal character of the process.

\section{Processes of alternating patterns with a double jump component}

Consider the sequence $\left\{T_{m}\right\}_{m \geq 0}$ of nonnegative independent random variables having distributions with the alternating density functions $f_{0}(t)$ and $f_{1}(t), t \geq 0$. Consider the flow of time instants $T^{+, m}=T_{0}+\ldots+T_{m-1}, m \geq 1, T^{+, 0}=0$.

Let $M=M(t)$ be the counting process,

$$
M(t)=\max \left\{m \geq 0 \mid T^{+, m} \leq t\right\}, \quad t>0 .
$$

The current state $\varepsilon_{i}(t), t \geq 0$, of the model is defined by

$$
\varepsilon_{0}(t)=\frac{1-(-1)^{M(t)}}{2}, \quad \varepsilon_{1}(t)=\frac{1+(-1)^{M(t)}}{2},
$$

such that $\varepsilon_{i}(0)=i, i \in\{0,1\}$.

Denote by $f_{0}^{(m)}(t), f_{1}^{(m)}(t)$ the conditional density functions of $T^{+, m}$ under the initial states $\varepsilon(0)=0$ and $\varepsilon(0)=1$ respectively. Functions $f_{i}^{(m)}, i \in\{0,1\}$, are defined by consecutive convolutions of densities $f_{0}$ and $f_{1}$ (beginning with $f_{i}$ ).

If the variables $T_{m}$ are i.i.d. exponentially distributed, $f_{0}(t) \equiv f_{1}(t)=$ $\mathrm{e}^{-\mu t} \mathbf{1}_{\{t>0\}}, \mu>0$, the sum $T^{+, m}$ is Erlang- $m$ distributed with the density function

$$
f^{(m)}(t)=\frac{\mu^{m} t^{m-1}}{(m-1) !} \mathrm{e}^{-\mu t}, \quad t>0 .
$$

In the case when $T_{m}$ are exponentially distributed and independent (with alternating intensities $\mu_{0}$ and $\left.\mu_{1}\right)$ the distributions $f_{i}^{(m)}(t), t>0$, are also known:

$$
\begin{aligned}
& f_{0}^{(m)}(t)=\mu_{0}^{(\times, m)} \cdot \frac{t^{m-1}}{(m-1) !} \exp \left(-\mu_{0} t\right) \Phi\left([m / 2] ; m ;\left(\mu_{0}-\mu_{1}\right) t\right), \\
& f_{1}^{(m)}(t)=\mu_{1}^{(\times, m)} \cdot \frac{t^{m-1}}{(m-1) !} \exp \left(-\mu_{1} t\right) \Phi\left([m / 2] ; m ;\left(\mu_{1}-\mu_{0}\right) t\right),
\end{aligned}
$$

where $\Phi(\cdot ; \cdot ; z)$ is the confluent hypergeometric Kummer function. Here [.] denotes the integer part and $\mu_{i}^{(\times, m)}$ is the consecutive product of $\mu_{i}$ and $\mu_{1-i}$ beginning with $\mu_{i}$, see Ratanov (2015, Proposition 2.1).

In this section we examine the jump-telegraph process $L(t)+r(t), t \geq 0$, which successively follows two alternating patterns during exponentially distributed time epochs $T_{m}$, i. e., for $t \in\left[T^{+, m}, T^{+, m}+T_{m}\right), m \geq 0$,

$$
L(t)=L_{m}(t), \quad r(t)=r_{m}(t),
$$


where $L_{m}, r_{m}, m \geq 0$, are independent processes, defined by (2.4) and (2.37) respectively.

More precisely, we consider the sequence $\tau_{m, n}, m, n \geq 0$, of independent exponentially distributed, $\operatorname{Exp}\left(\lambda_{m, n}\right), \lambda_{m, n}>0$, random variables. Let $N_{m}(t), t \geq 0$, $m \geq 0$, be (independent) Poisson processes, counting the arrivals of $\tau_{m, k}, k \geq 0$,

$$
N_{m}(t)=\max \left\{n \mid \sum_{k=0}^{n-1} \tau_{m, k} \leq t\right\} .
$$

Consider the continuous,

$$
L(t)=\sum_{m=0}^{M(t)-1} L_{m}\left(T_{m}\right)+L_{M(t)}\left(t-T^{+, M(t)}\right),
$$

and compound Poisson,

$$
r(t)=\sum_{m=0}^{M(t)-1} r_{m}\left(T_{m}\right)+r_{M(t)}\left(t-T^{+, M(t)}\right),
$$

random processes. Here

$$
L_{m}(t)=\int_{0}^{t} c_{m, N_{m}(u)} \mathrm{d} u=\sum_{n=0}^{N_{m}(t)-1} c_{m, n} \tau_{m, n}+c_{m, N_{m}(t)}\left(t-\tau_{m}^{+, N_{m}(t)}\right)
$$

and

$$
r_{m}(t)=\int_{0}^{t} r_{m, N(u)} \mathrm{d} N_{m}(u)=\sum_{n=0}^{N_{m}(t)-1} r_{m, n}, \quad t \geq 0,
$$

where $c_{m, n}$ are constants, and $r_{m, n}$ are independent random variables. The sums in (3.5) and (3.6) can be considered as compound Poisson processes with Poisson subordinators studied by Di Crescenzo et al. (2015).

The process performs additional jumps that occur when changing patterns. Suppose that the jump amplitude depends on the number of switchings during the current pattern. So, the jump process $R=R(t)$ is defined by

$$
R(t)=\sum_{m=1}^{M(t)} R_{m}\left(N_{m}\left(T_{m}\right)\right),
$$

Here $R_{m}(n)$ are independent random variables, independent of the counting processes $N$ and $M$.

Assume that the patterns are changing alternately, i. e., for the initial state $i, i \in\{0,1\}, i=\varepsilon(0)$, let

$$
\begin{array}{cl}
\lambda_{2 m, n}=\lambda_{n}^{i}, & \lambda_{2 m+1, n}=\lambda_{n}^{1-i} ; \\
c_{2 m, n}=c_{n}^{i}, & c_{2 m+1, n}=c_{n}^{1-i} ; \\
R_{2 m}(n) \stackrel{D}{=} R^{i}(n), & R_{2 m+1}(n) \stackrel{D}{=} R^{1-i}(n) ; \\
r_{2 m, n} \stackrel{D}{=} r_{n}^{i}, & r_{2 m+1, n} \stackrel{D}{=} r_{n}^{1-i} ; \\
m \geq 0, n \geq 0 .
\end{array}
$$


Here $\stackrel{D}{=}$ denotes the equality in distribution. The distributions of all processes depending on the initial state could be expressed in terms of parameters introduced by (3.8).

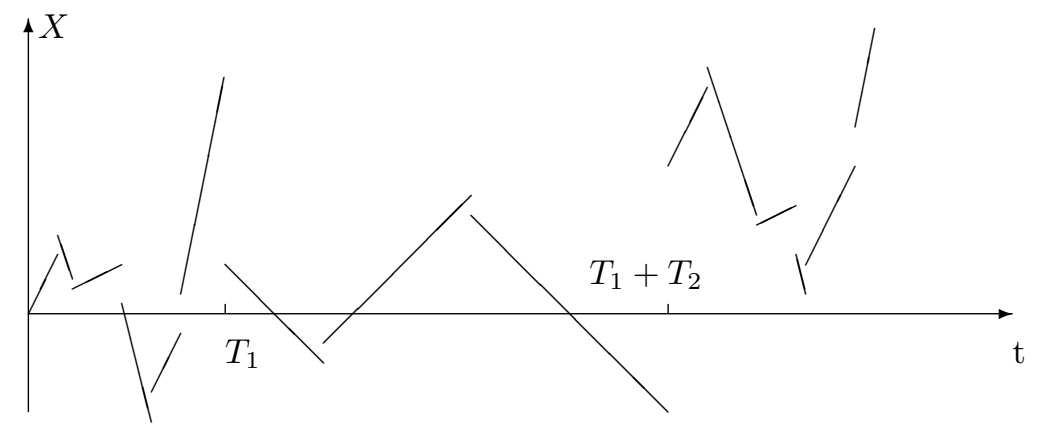

FiguRE 3.7. Sample path of $X=L(t)+r(t)+R(t)$.

We study the distribution of the sum

$$
X(t)=L(t)+r(t)+R(t), \quad t \geq 0 .
$$

Denote by $\mathbb{P}_{i}$ and $\mathbb{E}_{i}$ the conditional probability and the corresponding conditional expectation, if the initial pattern is given, $i=\varepsilon(0)$. The probability mass functions $\pi^{i}(t ; n)=\mathbb{P}\{N(t)=n \mid \varepsilon(0)=i\}, t \geq 0, i \in\{0,1\}$, follow equations (2.6),

$$
\frac{\mathrm{d}}{\mathrm{d} t} \pi^{i}(t ; n)=-\lambda_{n}^{i} \pi^{i}(t ; n)+\lambda_{n-1}^{i} \pi^{i}(t ; n-1), \quad i \in\{0,1\}
$$

By Theorem 2.10 we have

$$
\alpha_{i}(t):=\frac{\mathrm{d}}{\mathrm{d} t} \mathbb{E}[L(t)+r(t) \mid \varepsilon(0)=i]=\sum_{n=0}^{\infty}\left(c_{n}^{i}+\lambda_{n}^{i} \overline{r_{n}^{i}}\right) \pi^{i}(t ; n) .
$$

Let

$$
a_{i}(t)=\mathbb{E}\left[R^{i}(N(t)) \mid \varepsilon(0)=i\right]=\sum_{n=0}^{\infty} \overline{R^{i}(n)} \pi^{i}(t ; n), \quad i \in\{0,1\}, t \geq 0,
$$

assuming that the series in (3.11)-(3.12) converge. Here

$$
\overline{r_{n}^{i}}=\mathbb{E}\left[r_{n}^{i}\right], \quad \overline{R^{i}(n)}=\mathbb{E}\left[R^{i}(n)\right], \quad i \in\{0,1\},
$$

are the expectations of the jump amplitudes.

The (conditional) density functions $p^{X}=p_{i}^{X}(x, t ; m)$ of $X(t) \cdot \mathbf{1}_{\{M(t)=m\}}$ follow the integral equations

$$
\begin{gathered}
p_{i}^{X}(x, t ; m)=\int_{0}^{t} f_{i}(u)\left[\int_{-\infty}^{\infty} p_{1-i}^{X}\left(x-y-a_{i}(u), t-u ; m-1\right) \phi^{i}(y ; u) \mathrm{d} y\right] \mathrm{d} u, \\
t>0, i \in\{0,1\}, m \geq 1,
\end{gathered}
$$

where $\phi^{i}$ is the density function of $L^{i}(t)+r^{i}(t)$ defined by (2.38). For $m=0$ the density function is already known, $p_{i}^{X}(x, t ; 0)=\overline{F_{i}}(t) \phi^{i}(x, t)$, where $\overline{F_{i}}(u)=$ $\int_{u}^{\infty} f_{i}(s) \mathrm{d} s$ are the survival functions of $T_{m}$.

Let

$$
\mathfrak{M}_{i}(t):=\mathbb{E}_{i}[X(t)]=\mathbb{E}[X(t) \mid \varepsilon(0)=i], \quad i \in\{0,1\},
$$


be the expectations of $X(t)$ under the fixed initial state $\varepsilon(0)=i$.

Assume conditions (2.39)-(2.40) to be hold:

$$
\sum_{n=0}^{\infty}\left(c_{n}^{i}+\lambda_{n}^{i} \overline{r_{n}^{i}}\right) \pi_{i}(t ; n)<\infty \quad t \geq 0,
$$

and

$$
\left(c_{n}^{i}-\lambda_{n}^{i} \rho_{n}^{i}\right) \pi_{i}(t ; n) \rightrightarrows 0, \quad t \geq 0,
$$

as $n \rightarrow \infty ; i \in\{0,1\}$.

Theorem 3.1. Functions $\mathfrak{M}_{i}(t), i \in\{0,1\}$, satisfy the following integral equations,

$$
\begin{aligned}
& \mathfrak{M}_{0}(t)=A_{0}(t)+\int_{0}^{t} f_{0}(u) \mathfrak{M}_{1}(t-u) \mathrm{d} u, \\
& \mathfrak{M}_{1}(t)=A_{1}(t)+\int_{0}^{t} f_{1}(u) \mathfrak{M}_{0}(t-u) \mathrm{d} u,
\end{aligned}
$$

where

$$
A_{i}(t)=\int_{0}^{t}\left[\overline{F_{i}}(u) \alpha_{i}(u)+f_{i}(u) a_{i}(u)\right] \mathrm{d} u, \quad i \in\{0,1\} .
$$

See the definitions of $\alpha_{i}$ and $a_{i}$ in (3.11) and (3.12).

Proof: By conditioning on the first pattern's switching one can obtain

$$
\begin{aligned}
\mathfrak{M}_{0}(t) & =\overline{F_{0}}(t) \mathbb{E}\left\{L_{0}(t)+r_{0}(t) \mid \varepsilon(0)=0\right\} \\
& +\int_{0}^{t} f_{0}(u)\left[\mathbb{E}\left\{L_{0}(u)+r_{0}(u)+R_{0}(N(u)) \mid \varepsilon(0)=0\right\}+\mathfrak{M}_{1}(t-u)\right] \mathrm{d} u, \\
\mathfrak{M}_{1}(t) & =\overline{F_{1}}(t) \mathbb{E}\left\{L_{0}(t)+r_{0}(t) \mid \varepsilon(0)=1\right\} \\
& +\int_{0}^{t} f_{1}(u)\left[\mathbb{E}\left\{L_{0}(u)+r_{0}(u)+R_{0}(N(u)) \mid \varepsilon(0)=1\right\}+\mathfrak{M}_{0}(t-u)\right] \mathrm{d} u .
\end{aligned}
$$

Integrating by parts we get

$$
\begin{aligned}
\mathfrak{M}_{0}(t)=\int_{0}^{t} \overline{F_{0}}(u) \frac{\mathrm{d}}{\mathrm{d} u} \mathbb{E}_{0}\left\{L_{0}(u)+r_{0}(u)\right\} \mathrm{d} u & +\int_{0}^{t} f_{0}(u) a_{0}(u) \mathrm{d} u \\
& +\int_{0}^{t} f_{0}(u) \mathfrak{M}_{1}(t-u) \mathrm{d} u, \\
\mathfrak{M}_{1}(t)=\int_{0}^{t} \overline{F_{1}}(u) \frac{\mathrm{d}}{\mathrm{d} u} \mathbb{E}_{1}\left\{L_{0}(u)+r_{0}(u)\right\} \mathrm{d} u & +\int_{0}^{t} f_{1}(u) a_{1}(u) \mathrm{d} u \\
& +\int_{0}^{t} f_{1}(u) \mathfrak{M}_{0}(t-u) \mathrm{d} u .
\end{aligned}
$$

This gives (3.17)-(3.18).

Theorem 3.2. Let function $\alpha_{i}$ and $a_{i}$ be defined by (3.11)-(3.12).

- Let $a_{i} \neq 0, \frac{\alpha_{i}(t)}{a_{i}(t)}<0, \quad \forall t>0$, and

$$
\int_{0}^{\infty} \frac{\alpha_{i}(t)}{a_{i}(t)} \mathrm{d} t=-\infty
$$


If the alternating distributions of $T_{m}$ are defined by the survival functions

$$
\overline{F_{i}}(t)=\exp \left(\int_{0}^{t} \frac{\alpha_{i}(u)}{a_{i}(u)} \mathrm{d} u\right), \quad t \geq 0, \quad i \in\{0,1\},
$$

then $X=X(t)$ is the martingale.

- Let $R^{i}(n) \equiv 0$ and $c_{n}^{i} / \overline{r_{n}^{i}}<0, \forall n, i \in\{0,1\}$.

If the velocity switchings occur with the intensities $\lambda_{n}^{i}=-c_{n}^{i} / r_{n}^{i}, \forall n$, $i \in\{0,1\}$, then $X=X(t)$ is the martingale.

Proof: Due to (3.17)-(3.18) the renewal process $X$ is the martingale if and only if $A_{i}(t) \equiv 0, i \in\{0,1\}$, see $(3.19)$.

If $a_{i}(t) \neq 0, t>0$, then $A_{i} \equiv 0$ is equivalent to the set of identities

$$
\frac{f_{i}(t)}{\overline{F_{i}}(t)}=-\frac{\alpha_{i}(t)}{a_{i}(t)}, \quad t \geq 0, i \in\{0,1\} .
$$

By definition we have ${\overline{F_{i}}}^{\prime}(t)=-f_{i}(t), \quad \overline{F_{i}}(0)=1$. Hence (3.24) holds.

If $R^{i}(n) \equiv 0$ and $c_{n}^{i} / \overline{r_{n}^{i}}<0, \forall n$, then $A_{i} \equiv 0, i \in\{0,1\}$, is equivalent to $\alpha_{i} \equiv 0, i \in\{0,1\}$. The latter means that $\lambda_{n}^{i}=-c_{n}^{i} / \overline{r_{n}^{i}} i \in\{0,1\}, n \geq 0$.

Corollary 3.3. Let the elapsed time $T_{m}$ be exponentially distributed with parameters $\mu^{0}, \mu^{1}>0$, and $c_{n}^{i}+\lambda_{n}^{i} \overline{r_{n}^{i}}+\mu^{i} \overline{R_{n}^{i}}=0, i \in\{0,1\}$.

Then process $X=X(t)$ is the martingale.

\section{Market model}

Let process $X=X^{i}$ be defined by (3.9) and the initial state is given, $\varepsilon(0)=i$. Let $T_{m}, m \geq 0$, be elapsed times having exponential distributions with alternating parameters $\mu_{0}$ and $\mu_{1}$.

Assume that the market follows two possible (alternating) patterns, $\left\langle c_{n}^{0}, r_{n}^{0}, \lambda_{n}^{0}\right\rangle_{n \geq 0}$ and $\left\langle c_{n}^{1}, r_{n}^{1}, \lambda_{n}^{1}\right\rangle_{n \geq 0}$, during the consecutive elapsed times $T_{m}$. The price of risky asset is given by stochastic exponential of $X, S^{i}(t):=\mathcal{E}_{t}(X), t \geq 0$,

$$
S^{i}(t)=\mathcal{E}_{t}(X)=S_{0} \exp (L(t)) \prod_{n=0}^{N(t)}\left(1+r_{n}^{i}\right) \times \prod_{m=1}^{M(t)}\left(1+R_{m}^{i}\left(N_{m}\left(T_{m}\right)\right)\right) .
$$

The dynamics defined by (4.1) generalises the well-studied jump-telegraph model, Kolesnik and Ratanov (2013).

Model (4.1) can be interpreted as follows. Between time instants $T^{+, m}, m \geq 1$, market operates in the usual way. Further, at random times $T^{+, m}, m \geq 1$, a strategic investor (or regulator) provokes a price impact (of the amplitude $R_{m}\left(N_{m}\left(T_{m}\right)\right.$ )) accompanying by a pattern's switching. The amplitudes of jumps are assumed depending on the regulatory policy, as well as from the historical behaviour of the current pattern. Such behaviour of the strategic investor can be interpreted as a price manipulation strategy.

Note that if the regulator does not produce jumps of asset price, then the market able to hedge all risks; if $R_{m}^{i} \equiv 0$ and

$$
c_{n}^{i}+\lambda_{n}^{i} \overline{r_{n}^{i}}=0, \quad n \geq 0, i \in\{0,1\},
$$

then $S(t)$ is the martingale, see Theorem 3.2. Hence, the risk-neutral measure exists. 
In the case when the prices jump on $R^{i}(n)$ after regulation, where the jump amplitudes satisfy the inequality

$$
\frac{\mu^{i} \overline{R^{i}}(n)+c_{n}^{i}}{\overline{r_{n}^{i}}}<0, \quad n \geq 0, i \in\{0,1\},
$$

then the market is still free of arbitrage, see Theorem 3.2, and cf Kolesnik and Ratanov (2013).

If inequality (4.3) does not hold, then the risk-neutral measures do not exist and the risk of such behaviour should be considered as an inherent risk.

A certain policy of the strategic investor could trigger the arbitrage.

\section{Appendix}

The following easy results are used in the proofs of Theorem 2.1 and Theorem 2.8 .

Let functions

$$
\psi_{n}(z)=\frac{\mathrm{e}^{A z}}{(B-z)^{n+1}}, \quad n \geq 0,
$$

where $A, B$ are real numbers, be defined in the neighbourhood of $0,|z|<\varepsilon$. Hence, if $B>0$ we assume $z<B$, and if $B<0$, function $\psi_{n}$ is considered for $z>B$.

Proposition 1. The inverse Laplace transform $p_{n}=p_{n}(x)$ of $\psi_{n}$, defined by

$$
\int_{-\infty}^{\infty} \mathrm{e}^{z x} p_{n}(x) \mathrm{d} x=\psi_{n}(z), \quad|z|<\varepsilon,
$$

can be expressed as

$$
p_{n}(x)=\phi_{A, B}(x) \frac{(x-A)^{n}}{n !} \mathrm{e}^{B(A-x)} .
$$

Here

$$
\phi_{A, B}(x)=\left\{\begin{aligned}
\mathbf{1}_{\{x>A\}}, & \text { if } B>0, \\
-\mathbf{1}_{\{x<A\}}, & \text { if } B<0 .
\end{aligned}\right.
$$

Proof: Formula (1) follows from

$$
\begin{aligned}
& \int_{A}^{\infty} \mathrm{e}^{z x}(x-A)^{n} \mathrm{e}^{B(A-x)} \mathrm{d} x=\mathrm{e}^{A z} n !(B-z)^{-n-1}, \quad B-z>0, \quad B>0 ; \\
& -\int_{-\infty}^{A} \mathrm{e}^{z x}(x-A)^{n} \mathrm{e}^{B(A-x)} \mathrm{d} x=\mathrm{e}^{A z} n !(B-z)^{-n-1}, \quad B-z<0, \quad B<0 ;
\end{aligned}
$$

for $n \geq 0$.

Let functions

$$
\psi_{m}(z)=\frac{\mathrm{e}^{A z}}{(B+z)^{m+1}}, \quad m \geq 0,
$$

be defined for $z<-B$ and $A>0$.

Proposition 2. The inverse Laplace transform $g_{m}(t)$, defined by

$$
\int_{0}^{\infty} \mathrm{e}^{z t} g_{m}(t) \mathrm{d} t=\psi_{m}(z)
$$

can be expressed as

$$
g_{m}(t)=-\frac{(A-t)^{m}}{m !} \mathrm{e}^{B(t-A)} \mathbf{1}_{\{t>A\}} .
$$


Proof: The proof is similar to the proof of Proposition A1. It is based on the equality

$$
-\int_{A}^{\infty} \mathrm{e}^{z t}(A-t)^{m} \mathrm{e}^{B(t-A)} \mathrm{d} t=\mathrm{e}^{A z} m !(B+z)^{-m-1}, \quad B+z<0 .
$$

Proposition 3. Let $\Pi_{n}(x):=\left\{\vec{y}=\left(y_{0}, \ldots, y_{n-1}\right) \mid \sum_{i=0}^{n-1} y_{i}<x\right\} \subset \mathbb{R}^{n}$ and $\alpha_{0}, \ldots \alpha_{n}$ are positive and distinct constants. Therefore,

$$
\int_{\Pi_{n}(x)} \exp \left(-\sum_{k=0}^{n-1} y_{k}\left(\alpha_{k}-\alpha_{n}\right)\right) \mathrm{d} \vec{y}=\mathrm{e}^{\alpha_{n} x} \sum_{k=0}^{n} \kappa_{n, k}(\vec{\alpha}) \mathrm{e}^{-\alpha_{k} x} .
$$

Proof: See Ratanov (2014, (3.5)).

\section{References}

A. Alfonsi and P. Blanc. Dynamic optimal execution in a mixed-market-impact Hawkes price model. Finance Stoch. 20 (1), 183-218 (2016). MR3441291.

E. Bacry, I. Mastromatteo and J.-F. Muzy. Hawkes processes in finance. Market Microstructure and Liquidity 1 (1), 1550005 (2015). DOI: $10.1142 /$ S2382626615500057.

O. L. V. Costa and M. H. A. Davis. Impulse control of piecewise-deterministic processes. Math. Control Signals Systems 2 (3), 187-206 (1989). MR997213.

M. H. A. Davis. Piecewise-deterministic Markov processes: a general class of nondiffusion stochastic models. J. Roy. Statist. Soc. Ser. B 46 (3), 353-388 (1984). MR790622.

A. De Gregorio. Transport processes with random jump rate. Statist. Probab. Lett. 118, 127-134 (2016). MR3531493.

A. Di Crescenzo. On random motions with velocities alternating at Erlangdistributed random times. Adv. in Appl. Probab. 33 (3), 690-701 (2001). MR1860096.

A. Di Crescenzo and B. Martinucci. A damped telegraph random process with logistic stationary distribution. J. Appl. Probab. 47 (1), 84-96 (2010). MR2654760.

A. Di Crescenzo, B. Martinucci and S. Zacks. Compound Poisson process with a Poisson subordinator. J. Appl. Probab. 52 (2), 360-374 (2015). MR3372080.

A. Di Crescenzo and F. Pellerey. On prices' evolutions based on geometric telegrapher's process. Appl. Stoch. Models Bus. Ind. 18 (2), 171-184 (2002). MR1907356.

G. B. Di Mazi, Yu. M. Kabanov and V. I. Runggal/der. Mean-square hedging of options on a stock with Markov volatilities. Teor. Veroyatnost. i Primenen. 39 (1), 211-222 (1994). MR1348196.

P. Embrechts, T. Liniger and L. Lin. Multivariate Hawkes processes: an application to financial data. J. Appl. Probab. 48A (New frontiers in applied probability: a Festschrift for Søren Asmussen), 367-378 (2011). MR2865638.

W. Feller. An Introduction to Probability Theory and Its Applications. Vol. I. John Wiley \& Sons, Inc., New York, N.Y. (1950). MR0038583. 
S. K. Foong. First-passage time, maximum displacement, and Kac's solution of the telegrapher equation. Phys. Rev. A (3) 46 (2), R707-R710 (1992). MR1175578.

S. K. Foong and S. Kanno. Properties of the telegrapher's random process with or without a trap. Stochastic Process. Appl. 53 (1), 147-173 (1994). MR1290711.

B. V. Gnedenko and I. N. Kovalenko. Introduction to queueing theory. Translated from Russian by R. Kondor. Translation edited by D. Louvish. Israel Program for Scientific Translations, Jerusalem; Daniel Davey \& Co., Inc., Hartford, Conn. (1968). MR0240884.

S. Goldstein. On diffusion by discontinuous movements, and on the telegraph equation. Quart. J. Mech. Appl. Math. 4, 129-156 (1951). MR0047963.

A. G. Hawkes. Spectra of some self-exciting and mutually exciting point processes. Biometrika 58, 83-90 (1971). MR0278410.

M. Kac. A stochastic model related to the telegrapher's equation. Rocky Mountain J. Math. 4, 497-509 (1974). MR0510166.

A. Kolesnik. The equations of Markovian random evolution on the line. J. Appl. Probab. 35 (1), 27-35 (1998). MR1622442.

A. D. Kolesnik and N. Ratanov. Telegraph processes and option pricing. SpringerBriefs in Statistics. Springer, Heidelberg (2013). ISBN 978-3-642-40525-9; 978-3642-40526-6. MR3115087.

Yu. I. Kuznetsov. Матрицы и многочлены. Цхаст/ I. Rossiǔskaya Akademiya Nauk Sibirskoe Otdelenie, Institut Vychislitel/nol̆ Matematiki i Matematicheskou Geofiziki, Novosibirsk (2003). MR2031223.

O. López and N. Ratanov. Option pricing under jump-telegraph model with random jumps. J. Appl. Prob. 49 (3), 838-849 (2012).

O. López and N. Ratanov. On the asymmetric telegraph processes. J. Appl. Probab. 51 (2), 569-589 (2014). MR3217786.

E. Orsingher. Motions with reflecting and absorbing barriers driven by the telegraph equation. Random Oper. Stochastic Equations 3 (1), 9-21 (1995). MR1326804.

E. Orsingher and B. Bassan. On a $2 n$-valued telegraph signal and the related integrated process. Stochastics Stochastics Rep. 38 (3), 159-173 (1992). MR1274901.

M. A. Pinsky. Lectures on random evolution. World Scientific Publishing Co., Inc., River Edge, NJ (1991). ISBN 981-02-0559-7. MR1143780.

N. Ratanov. Telegraph processes and option pricing. 2nd Nordic-Russian Symposium on Stochastic Analysis, Beitostolen, Norway (1999).

N. Ratanov. A jump telegraph model for option pricing. Quant. Finance 7 (5), 575-583 (2007). MR2358921.

N. Ratanov. Option pricing model based on a Markov-modulated diffusion with jumps. Braz. J. Probab. Stat. 24 (2), 413-431 (2010). MR2643573.

N. Ratanov. On piecewise linear processes. Statist. Probab. Lett. 90, 60-67 (2014). MR3196858.

N. Ratanov. Hypo-exponential distributions and compound Poisson processes with alternating parameters. Statist. Probab. Lett. 107, 71-78 (2015). MR3412757.

İ. V. Samollenko. Damping Markov random evolution. Ukraïn. Mat. Zh. 54 (3), 364-372 (2002). MR1952795.

B. de Saporta and F. Dufour. Numerical method for impulse control of piecewise deterministic Markov processes. Automatica J. IFAC 48 (5), 779-793 (2012). MR2912800. 
D. L. Snyder and M. I. Miller. Random Point Processes in Time and Space. Springer, New York-Berlin-Heidelberg-London (1991).

W. Stadje and S. Zacks. Telegraph processes with random velocities. J. Appl. Probab. 41 (3), 665-678 (2004). MR2074815.

G. I. Taylor. Diffusion by Continuous Movements. Proc. London Math. Soc. S220 (1), 196 (1922). MR1577363.

S. Zacks. Generalized integrated telegraph processes and the distribution of related stopping times. J. Appl. Probab. 41 (2), 497-507 (2004). MR2052587. 